\title{
Comparison of transcriptional profiles of Clostridium thermocellum grown on cellobiose and pretreated yellow poplar using RNA-Seq
}

\author{
Hui Wei ${ }^{1 * \neq}$, Yan Fu ${ }^{2+\neq}$, Lauren Magnusson ${ }^{1}$, John O. Baker ${ }^{1}$, Pin-Ching Maness ${ }^{1}$, Qi Xu ${ }^{1}$, \\ Shihui Yang ${ }^{3}$, Andrew Bowersox ${ }^{1,3}$, Igor Bogorad ${ }^{1}$, Wei Wang ${ }^{1}$, Melvin P. Tucker ${ }^{3}$, Michael E. Himmel ${ }^{1}$ \\ and Shi-You Ding ${ }^{1 *}$
}

1 Biosciences Center, National Renewable Energy Laboratory, Golden, CO, USA

${ }^{2}$ Center for Plant Genomics, lowa State University, Ames, IA, USA

${ }^{3}$ National Bioenergy Center, National Renewable Energy Laboratory, Golden, CO, USA

\section{Edited by:}

Nigel Peter Minton, University of

Nottingham, UK

\section{Reviewed by:}

Patricia Coutinho Dos Santos, Wake

Forest University, USA

Marianne Guiral, Centre National de

la Recherche Scientifique, France

\section{*Correspondence:}

Hui Wei and Shi-You Ding, National Renewable Energy Laboratory,

Biosciences Center, 15013 Denver W Pkwy, Golden, CO 80401, USA

e-mail: hui.wei@nrel.gov;

shi.you.ding@nrel.gov

${ }^{\dagger}$ Present address:

Yan Fu, Monsanto Company,

St. Louis, USA

¥ These authors have contributed equally to this work.
The anaerobic, thermophilic bacterium, Clostridium thermocellum, secretes multi-protein enzyme complexes, termed cellulosomes, which synergistically interact with the microbial cell surface and efficiently disassemble plant cell wall biomass. $C$. thermocellum has also been considered a potential consolidated bioprocessing (CBP) organism due to its ability to produce the biofuel products, hydrogen, and ethanol. We found that $C$. thermocellum fermentation of pretreated yellow poplar (PYP) produced 30 and $39 \%$ of ethanol and hydrogen product concentrations, respectively, compared to fermentation of cellobiose. RNA-seq was used to analyze the transcriptional profiles of these cells. The PYP-grown cells taken for analysis at the late stationary phase showed 1211 genes up-regulated and 314 down-regulated by more than two-fold compared to the cellobiose-grown cells. These affected genes cover a broad spectrum of specific functional categories. The transcriptional analysis was further validated by sub-proteomics data taken from the literature; as well as by quantitative reverse transcription-PCR (qRT-PCR) analyses of selected genes. Specifically, 47 cellulosomal protein-encoding genes, genes for 4 pairs of Sigl-Rsgl for polysaccharide sensing, 7 cellodextrin $A B C$ transporter genes, and a set of $\mathrm{NAD}(\mathrm{P}) \mathrm{H}$ hydogenase and alcohol dehydrogenase genes were up-regulated for cells growing on PYP compared to cellobiose. These genes could be potential candidates for future studies aimed at gaining insight into the regulatory mechanism of this organism as well as for improvement of $C$. thermocellum in its role as a CBP organism.

Keywords: Clostridium thermocellum, transcriptomics, RNA-Seq, pretreated yellow poplar (PYP), cellobiose, cellulosome, ethanol, hydrogen

\section{INTRODUCTION}

Microbial conversion of biomass to biofuels is an attractive route for biofuel development, but an essential challenge is to increase the microbial capacity both for overcoming the biomass recalcitrance and for converting the biomass-derived sugars to biofuels (Himmel et al., 2007; Alper and Stephanopoulos, 2009). Clostridium thermocellum, a gram-positive, thermophilic, anaerobic bacterium, is one of the model consolidated bioprocessing (CBP) systems used to study the enzymatic hydrolysis of cellulosic biomass to produce fuels (Islam et al., 2006; Brown et al., 2012; Yang et al., 2012). The following features of C. thermocellum

\footnotetext{
Abbreviations: Abf, $\alpha$-N-arabinofuranosidase; ADH, alcohol dehydrogenase; $\mathrm{CBO}$, cellobiose-grown cells only; Cbp, carbohydrate-binding protein; CBP, consolidated bioprocessing; CDP, cellodextrin phosphorylase; CEP, cellobiose phosphorylases; COG, clusters of orthologous groups; emPAI, exponentially modified protein abundance index; FC, fold changes; Fdred, reduced ferredoxin; GH, glycoside hydrolases; NSAF, normalized spectral abundance factor; $P F$, passing filter; PTA, phosphotransacetylase; PYP, pretreated yellow poplar; PYPO, PYP-grown cells only; $q R T-P C R$, quantitative reverse transcription-PCR; RPKM, reads per kilobase of exon model per million mapped reads; RsgI, RsgI-like anti-sigma factors; SigI, sigma factor.
}

contribute to its suitability as a model cellulolytic, biofuelproducing bacterium: (1) It produces cellulosomes, a type of highly organized multi-protein enzyme complexes, which have been shown to be highly efficient enzyme systems in deconstructing plant cell wall, especially in degrading the recalcitrant substrate crystalline cellulose (Bayer et al., 1998, 2004). (2) It carries out mixed-product fermentation, producing ethanol, $\mathrm{H}_{2}$ and numerous organic acids including acetate, formate, and lactate (Demain et al., 2005; Islam et al., 2006). (3) It is suitable for both submerged and solid-state fermentation (Bayer et al., 2007), the latter having similarity to compost in the set-up of feedstock (Wei et al., 2012). (4) Its genome sequence is available (http:// genome.jgi-psf.org/cloth/cloth.info.html) and many of the cellulolytic enzymes are identified and biochemically characterized. The knowledge gained from studies of this species will benefit work on other clostridial species of industrial interest, such as C. acetobutylicum, known to produce the potential fuels acetone, ethanol, and butanol (Cooksley et al., 2012).

Fulfilling this potential will require a more in-depth understanding of the metabolic and genetic mechanisms by which 
C. thermocellum utilizes recalcitrant biomass substrate. So far, a number of studies have been carried out on $C$. thermocellum, such as transcriptomic analysis of stress responses to ethanol, furfural, and heat during growth on pure sugars (i.e., cellobiose) (Yang et al., 2012), time course studies of cell growth on pure crystalline cellulose (Avicel) (Raman et al., 2011), and comparisons of growth on pure crystalline cellulose (Sigmacel 50) and cellobiose (Riederer et al., 2011). However, despite reports on the sub-proteomic analyses of cellulosomes from $C$. thermocellum grown on Avicel (Gold and Martin, 2007) and on combinations of Avicel with pectin and/or xylan, or on pretreated switchgrass (Raman et al., 2009), there were no genome-wide transcriptomic studies reported for growth on pretreated woody plant biomass until most recently a transcriptomic analysis comparing C. thermocellum cells grown on pretreated switchgrass and woody biomass, cottonwood (Populus trichocarpa $\times$ Populus deltoids; black cottonwood $\times$ eastern cottonwood in common names), was published on December 2, 2013 (Wilson et al., 2013a), after the submission of this manuscript.

Woody biomass has been found to be more recalcitrant to enzyme digestion than is herbaceous biomass. For example, whereas the Recalcitrance Index for switchgrass is $\sim 0.35$, the index for hardwoods, such as yellow poplar, is 0.56 , indicating that hardwoods are more difficult to be degraded (Wei et al., 2009). Previous studies have shown that cellulolytic bacteria grown on different lignocellulosic substrates have different levels of glycoside hydrolase (GH) activities (Irwin et al., 2003). As such C. thermocellum grown on pretreated woody plant biomass is likely to have distinctive responsive genes, as well as different composition of cellulolytic enzymes different from those grown on herbaceous biomass. Recently, we found that species of the genus Clostridium (including $C$. thermocellum) were among the dominant species, comprising $6.3 \%$ of the total, in an anaerobic community decomposing yellow poplar wood chips (van der Lelie et al., 2012). In parallel, we studied the yellow poplar compost system (Wei et al., 2012), in which Clostridium was also found to be one of the dominant bacteria (data not shown). These results prompted us to explore $C$. thermocellum grown on dilute acid-pretreated yellow poplar (PYP) as a single-species model for the plant cell wall-degradation. PYP is a widely-utilized feedstock in process-development for conversion of biomass to fuels and chemicals, and it is important to identify specific enzymes that this organism calls into action to attack this form of the feedstock/substrate.

PYP has been previously demonstrated that $60 \%$ conversion to simple sugars can be achieved with a loading of $\sim 8.4 \mathrm{mg}$ per $\mathrm{g}$ biomass cellulose of a mixture of Trichoderma reesei CBHI and Acidothermus cellulolyticus E1 (95\%: 5\% on molar basis) (Vinzant et al., 1994; Baker et al., 1997). In this study, C. thermocellum was bench fermented using PYP and cellobiose as sole carbon sources, respectively, and transcriptional profiles were analyzed. RNA-seq is a recently developed technology for transcriptome profiling, which uses next-generation sequencing to reveal the presence and quantity of RNA in biological samples. The goals of this study are two-fold: first, we identified genes responsive for degradation of recalcitrant biomass substrates in PYP- vs. cellobiose-grown C. thermocellum cells. Secondly, we specifically focused on candidate genes related to cellulosome, cellodextrin transport, polysaccharide signal transduction and end-product synthesis related genes. These candidate genes are likely to be valuable for mechanism study; as well as for protein-engineering to further improve the abilities of this already potent organism to degrade the cell walls of recalcitrant biomass feedstocks.

\section{MATERIALS AND METHODS}

Figure 1A shows the overall experimental approach we designed to investigate the $C$. thermocellum utilization of pretreated biomass substrates as reflected by changes in the cell's transcriptome. Details of the experimental approach are described in the following sections.

\section{CARBON AND LIGNOCELLULOSIC SUBSTRATES}

Cellobiose and other chemical compounds were purchased from Sigma (St. Louis, MO). PYP was prepared as described previously (Tucker et al., 1998). Briefly, the milled yellow biomass (20\% solids loading) was pretreated in $0.21 \% \mathrm{w} / \mathrm{w} \mathrm{H}_{2} \mathrm{SO}_{4}$ at $200^{\circ} \mathrm{C}$ for 4 min. The resultant PYP contained $\sim 65 \%$ cellulose, $4 \%$ xylan, and 31\% lignin (dry weight basis). PYP was exhaustively washed with deionized water until the $\mathrm{pH}$ reached that of deionized water, prior to being used in medium preparation as described below.

\section{MICROORGANISMS AND CULTURE CONDITIONS}

C. thermocellum ATCC 27405 was routinely cultured at $60^{\circ} \mathrm{C}$ anaerobically in $30-\mathrm{mL}$ serum bottles containing $10 \mathrm{~mL}$ of ATCC medium 1191 containing cellobiose, and was subcultured with $2 \%$ inoculum taken at exponential growth phase. For this study, similarly to practices in the literature (Gold and Martin, 2007; Rydzak et al., 2009), 100-mL batch culture in 250-mL serum bottles was used for the growth of the strain anaerobically at $58^{\circ} \mathrm{C}$ in ATCC medium 1191, containing $0.30 \%(\mathrm{w} / \mathrm{v})$ cellobiose as the control carbohydrate substrate, or $0.44 \%$ PYP as biomass substrate (sugar-content equivalent to $0.30 \%$ cellobiose) with an agitation of $130 \mathrm{rpm}$. Cell growth was monitored based on either the measurement of optical density (OD) by spectrophotometry at $600 \mathrm{~nm}\left(\mathrm{OD}_{600}\right)$ for cellobiose-grown culture, or the increase in pellet protein amount for PYP-grown culture using the method described in literature (Raman et al., 2009). Three independent sets of PYP- vs. cellobiose-grown cell cultures, harvested at the late stationary phase $(20 \mathrm{~h}$ for cellobiose-grown cells and $36 \mathrm{~h}$ for PYP-grown cells), were carried through the downstream RNA extraction preparations. Two sets of RNA preparations were used for cDNA library construction and RNA-Seq, whereas all three sets of RNA preparations were used for qRT-PCR analysis in verifying RNA-Seq data of selected genes.

\section{MEASUREMENT OF $\mathrm{H}_{2}$ AND ETHANOL, AND ISOLATION OF BACTERIAL TOTAL RNA}

Cells were harvested for RNA isolation in late stationary phase after the hydrogen concentration at the headspace was analyzed using GC method, wherein an Agilent 7890A GC equipped with a Supelco 60/80 molsieve 5A column was used for the measurement of $\mathrm{H}_{2}$. The cell culture was centrifuged at high speed $(8000 \times \mathrm{g})$ for $5 \mathrm{~min}$ to pellet the bacterial cells. Cell-free culture supernatants were used to measure ethanol concentration by 


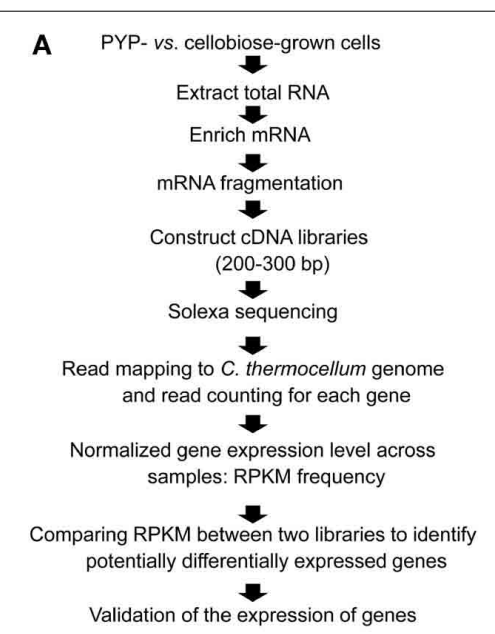

B

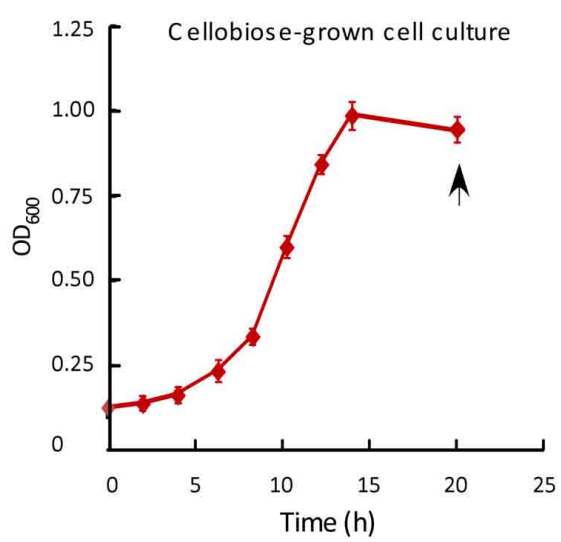

C

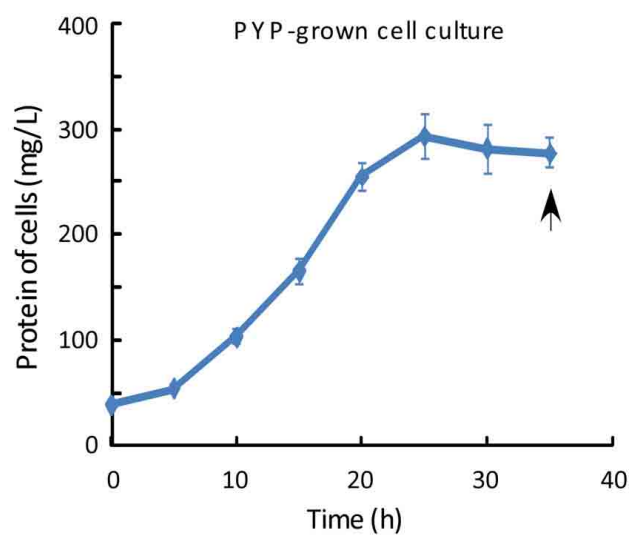

FIGURE 1 | Flowchart for experimental procedures, along with growth curves for cellobiose- and PYP-grown $C$. thermocellum cells. (A) Flowchart outlining the experimental procedures; RPKM, reads per kilobase of exon model per million mapped reads. (B) $\mathrm{OD}_{600}$ for cellobiose-grown cells was measured by spectrophotometry at $600 \mathrm{~nm}$
$\left(\mathrm{OD}_{600}\right)$. (C) Cell pellet protein concentration for pretreated yellow poplar (PYP)-grown cell culture. The doubling time for cellobiose- and PYP-grown cells was 3.3 and $7.8 \mathrm{~h}$, respectively. Data are presented as the mean $( \pm S E)$ of 3 replicates. Arrows indicate the time points sampled for transcriptomic analyses. using HPLC (Agilent) with a refractive index detector (Shimadzu, Kyoto, Japan). All samples were filtered through a $0.45-\mu \mathrm{m}$ filter before HPLC analysis. The organic acids were separated in an Aminex HPX-87H column (Bio-Rad) running at a flow rate of $0.6 \mathrm{ml} / \mathrm{min}$ at $50^{\circ} \mathrm{C}$, with $4 \mathrm{mM} \mathrm{H}_{2} \mathrm{SO}_{4}$ as eluent.

The cell pellets collected from $10 \mathrm{~mL}$ culture were used for RNA extraction, using a combined protocol (Yang et al., 2012), in which the TRIzol (Invitrogen, Carlsbad, CA) extraction aqueous phase was mixed with an equal volume of $70 \%$ ethanol, and then applied to the column of Qiagen RNeasy Mini Kit (Qiagen, Valencia, CA) for purification according to the manufacturer's instructions to obtain total RNAs. $7.5 \mu \mathrm{g}$ total RNAs were mixed with $3 \mu \mathrm{L}$ DNase I (Invitrogen; $1 \mathrm{U} / \mu \mathrm{L}$ ) in $3 \mu \mathrm{L} 10 \mathrm{x}$ DNase I reaction buffer, with adding RNase-free water added up to a total volume of $30 \mu \mathrm{L}$, and incubated at room temperature for $15 \mathrm{~min}$. The DNase I was inactivated by adding $3 \mu \mathrm{L}$ of $25 \mathrm{mM}$ EDTA and heating for $10 \mathrm{~min}$ at $65^{\circ} \mathrm{C}$. The DNase I treated total RNA was precipitated by the ethanol-glycogen method and redissolved in $15 \mu \mathrm{L}$ of $1 \mathrm{mM}$ EDTA. Since the total RNAs contains 75\% rRNA (Chen and Duan, 2011), mRNA was enriched by using the MICROBExpress Bacterial mRNA Enrichment Kit (cat \# AM1905, Ambion, Life Technologies, Austin, TX) to remove 16 and 23 s rRNAs. The resultant RNAs were quantified and analyzed for integrity on the Agilent 2100 Bioanalyzer, and used for cDNA library construction as described below.

\section{CDNA LIBRARY CONSTRUCTION, SEQUENCING, AND READ MAPPING}

cDNA libraries were constructed with the RNA-Seq sample preparation kit (RS-100-0801, Illumina, San Diego, CA), using the procedure provided by the manufacturer. Briefly, the above enriched mRNA samples were fragmented, and then annealed to random hexamers and reverse transcribed. After second strand synthesis, end repair, and A-tailing, cDNA fragment ends were ligated to adapters that were complementary to sequencing primers. Resultant cDNA libraries were size separated on agarose gels with $\sim 200 \mathrm{bp}$ fragments being excised, and amplified by 15 cycles of PCR. The prepared cDNA libraries were sequenced on an Illumina Genome Analyzer II by the Michigan State University DNA facility, using the standard protocols and running for 75 cycles of data acquisition. Solexa reads were aligned to the reference genome sequence of $C$. thermocellum strain (ATCC 27405) deposited at NCBI (Accession: NC_009012.1) using Bowtie (Langmead et al., 2009). The reference genome is $3.8-\mathrm{Mb}$ long with 3305 predicted genes, including 71 structural RNA genes, according to the NCBI website for the genome (http://www.ncbi.nlm.nih.gov/genome/? term=Clostridium+thermocellum) accessed on April 28, 2010. The best end-to-end alignment (ties broken by read quality) was used with no more than two mismatches. The reads that cannot be unambiguously mapped were not included for further analysis. The above alignment approach and criteria were similar to those used in literature (Jia et al., 2009; Lin et al., 2011).

\section{GENE EXPRESSION NORMALIZATION AND IDENTIFICATION OF DIFFERENTIALLY EXPRESSED GENES}

After read mapping, the midpoint of the read-reference alignment was used to determine which gene that read belongs to (or was derived from), and the RNA-seq read counts for each gene can be then calculated. To facilitate comparison of gene expression levels, both within and between two samples, we quantified and normalized gene expression levels by calculating the reads per kilobase of exon model per million mapped reads (RPKM), i.e., calculating the number of reads mapped per kilobase of exon model divided by the total number of mapped reads in the whole dataset (Mortazavi et al., 2008). As an indicator of gene expression level, the RPKM is widely recognized as a measure of Solexa read density that reflects the molar concentration of a transcript in the starting RNA sample, thus making the 
normalized gene expression levels comparable within and among samples.

The fold-change for the expression of an individual gene was calculated by taking the ratio of RPKM in PYP-grown cells to that in cellobiose-grown cells (RPKM $M_{P Y P} / R_{P K M}$ cellobiose $)$. The cutoff value for defining a gene as "differentially expressed" is either a two-fold increase (with the value of RPKM $M_{P Y P} / R_{P K M} M_{\text {cellobiose }}$ larger than 2.0), or a two-fold decrease (with the value of $\mathrm{RPKM}_{\mathrm{PYP}} / \mathrm{RPKM}_{\text {cellobiose }}$ less than 0.5).

\section{STATISTICAL ANALYSIS}

The log2-transformed raw RPKM dataset was imported into the statistical analysis software JMP Genomics 6.0 software (SAS Institute, NC), and the data of PYP- and cellobiose-grown cell samples were normalized together using the LOWESS normalization algorithm within JMP Genomics. To determine if the differential expression levels between PYP- and cellobiosegrown cell samples were statistically significant, the normalized $\log 2(\mathrm{RPKM})$ data were subjected to One-Way analysis of variance (ANOVA) as described in literature (Yang et al., 2012, 2013; Wilson et al., 2013b). The False Discovery Rate (FDR) testing method was used with a significance threshold of $p<0.05$ being considered statistically significant.

\section{BIOLOGICAL INTERPRETATION OF DIFFERENTIALLY EXPRESSED GENES}

The identified, differentially expressed genes were interpreted and discussed in the context of biological processes and functions, using clusters of orthologous groups (COG) and CarbohydrateActive enZYmes (CAZy) analyses of proteins (www.cazy.org/).

\section{QUANTITATIVE REVERSE TRANSCRIPTION-PCR (qRT-PCR)}

Based on their potential functional importance, 12 genes were selected for validating the results of the RNA-Seq analysis. The primers for these genes were either based on literature or designed with Primer Express 3.0 software (Applied Biosystems), and are described along with the PCR results in the Results and Discussion section. Total RNA was extracted from three sets of independent cultures grown on PYP vs. cellobiose as described above, and then converted to cDNA by random priming, using the SuperScript II kit (Invitrogen, San Diego, CA). PCR reactions were run in triplicate using procedure as previously described (Wei et al., 2012). The transcription level of genes was determined according to the $2^{-\Delta \Delta C T}$ method, using RecA as a reference gene for the normalization of gene expression levels (Stevenson and Weimer, 2005).

\section{CORRELATION ANALYSIS FOR CELLULOSOME-RELATED GENE EXPRESSION AND PROTEIN ABUNDANCE}

Two sets of quantitative cellulosomal protein data of cellobiosegrown C. thermocellum cells at late stationery phase were retrieved from literature (Gold and Martin, 2007; Raman et al., 2009), and plotted against our sub-dataset of $\log 2(\mathrm{RPKM})$ values of cellulosomal genes in cells grown on the same substrate at the same culture phase in this study. Pearson correlation coefficient values were calculated using Microsoft Excel (Microsoft Corporation, Redmond, WA, USA), and used as an indicator for the degree of correlation for the compared pairs.

\section{COMPOSITIONAL ANALYSIS OF PRETREATED YELLOW POPLAR RESIDUES}

The PYP residues from PYP-grown $C$. thermocellum culture were collected by centrifugation at low speed $(100 \times \mathrm{g})$ for $2 \mathrm{~min}$ to precipitate the insoluble substrate not consumed by the bacterial cells in the culture. Such centrifugation speed has been used in the literature to remove any insoluble substrate in C. thermocellum culture (Dror et al., 2005). Compositional analysis of the collected PYP residue was performed by the National Bioenergy Center, National Renewable Energy Laboratory, using method described in literature (Templeton et al., 2010).

\section{RESULTS AND DISCUSSION C. THERMOCELLUM GROWTH AND THE PRODUCTION OF HYDROGEN AND ETHANOL}

The first step of this study, as illustrated in Figures $\mathbf{1 A - C}$, is the growth of $C$. thermocellum ATCC 27405 with two types of carbohydrate substrates, PYP being compared with cellobiose. GC analysis of gas composition in the headspace of batch cultures at the late stationary phase revealed a production yield of 1.22 vs. 0.92 mole $\mathrm{H}_{2}$ /mole glucose consumed in cellobiose- and PYP-grown C. thermocellum, respectively (Table 1). Furthermore, HPLC analysis of metabolite production in supernatants of harvested cell culture revealed a production yield of 0.51 vs. 0.30 mole ethanol /mole glucose equivalent consumed in cellobioseand PYP-grown C. thermocellum, respectively. Overall, the production of hydrogen and ethanol by PYP-grown C. thermocellum are 75 and $58 \%$ of those by cellobiose-grown C. thermocellum, respectively (Table 1). Wet chemistry analysis of spent PYP solids revealed that $52 \%$ of the glucan in the starting PYP substrate was consumed during the fermentations, which can partially explain the lower absolute $\mathrm{H}_{2}$ and ethanol productions compared to the culture on cellobiose substrate (in which nearly all cellobiose was depleted at late stationary phase).

\section{RNA-Seq RESULTS}

The quality of RNA-Seq cDNA libraries was assessed on a Bioanalyzer prior to GA II (Figure 2). The results showed the size distribution of cDNA library was between 200 and 300 bp, which met the requirement for Solexa sequencing.

For the two PYP-grown cell cDNA library samples, Solexa sequencing from the first cDNA library generated 20.8 million total raw reads, with 14.5 million passing filter reads $(70 \% \mathrm{PF}$ reads); from the second cDNA library generated 19.6 million total raw reads, with 14.1 million passing filter reads (72\% PF reads). Out of these PYP-grown cell derived reads, 2.3 and 2.1 millions reads were unambiguously mapped to $C$. thermocellum genome with the criteria set in the Materials and Methods section, respectively. For the two cellobiose-grown cell cDNA library samples, Solexa sequencing from their first cDNA library generated 21.1 million total raw reads, with 15.7 million passing filter reads $(74 \%$ PF reads); from the second cDNA library generated 19.7 million total raw reads, with 14.0 million passing filter reads $(71 \%$ $\mathrm{PF}$ reads). Out of these cellobiose-grown cell-derived reads, 2.6 and 2.2 millions reads were unambiguously mapped to $C$. thermocellum genome, respectively. The RPKM value for each gene in each condition was calculated as RPKM = Reads number 
Table 1 | Hydrogen, ethanol and acetate production at the late stationary phase of $C$. thermocellum culture.

\begin{tabular}{lccc}
\hline Cell culture & \multicolumn{2}{c}{ Yields } & Acetate/Ethanol \\
\cline { 2 - 3 } & Hydrogen & Ethanol & Acetate \\
\cline { 2 - 4 } & (Mole product/Mole glucose equivalent consumed) \\
\hline Cellobiose-grown cells & $1.22 \pm 0.11$ & $0.51 \pm 0.05$ & $0.55 \pm 0.07$ \\
PYP-grown cells & $0.92 \pm 0.09$ & $0.30 \pm 0.04$ & $0.27 \pm 0.04$ \\
Yield in PYP-grown cells/yield in cellobiose-grown cells (\%) & 75 & 58 & 49
\end{tabular}

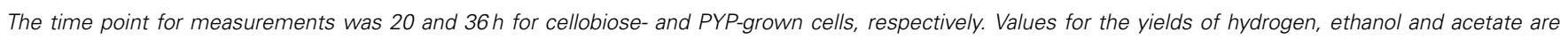
means \pm s.e.m. of three independent experiments. PYP, acid-pretreated yellow poplar.

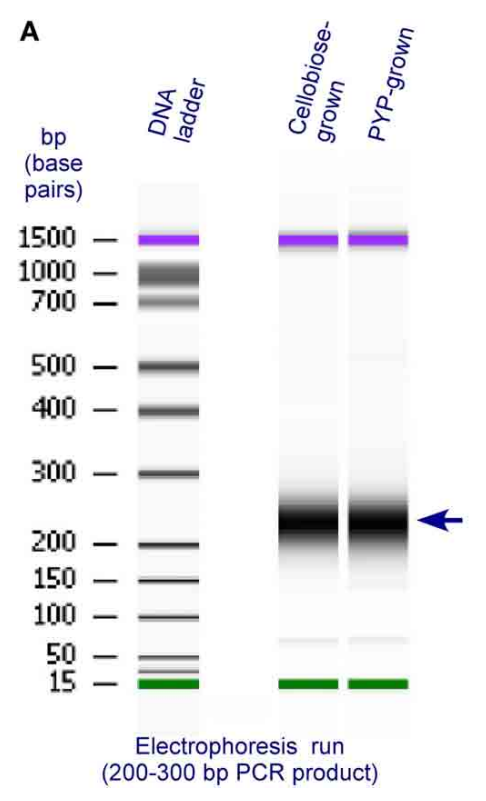

FIGURE 2 | Validation and quantification of representative, final RNA-Seq cDNA libraries prior to GA II, using an Agilent 2100 Bioanalyzer and the DNA 1000 Nano Chip Kit (Agilent, Technologies, Santa Clara, CA,

B
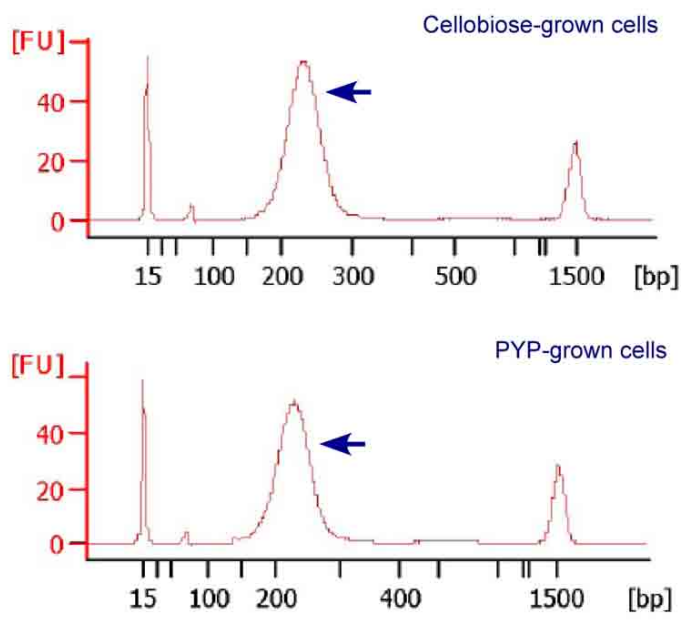

cDNA library bioanalyzer trace

USA). The RNA-Seq cDNA libraries were prepared from RNA extracted from C. thermocellum cells grown on cellobiose or PYP. (A) Electrophoresis run of the cDNA libraries by the Bioanalyzer; (B) bioanalyzer trace of cDNA libraries. mapped to gene/Length of the gene $(\mathrm{kb}) /$ Total reads number (million reads), as described in the Materials and Methods section.

Overall, sequence analysis successfully aligned the Solexa reads to 3081 protein-coding genes and 61 structural RNA genes in cellobiose- and/or PYP-grown C. thermocellum mRNA samples, accounting for $95 \%$ [i.e., $(3081+61) / 3305$ ] of all C. thermocellum genes in the genome, indicating that RNA-Seq analysis in this study achieved comprehensive coverage of the C. thermocellum transcriptome. In addition, the above result is consistent with the reports that in the genomes of other microorganisms such as Saccharomyces cerevisiae and S. pombe, more than $90 \%$ of the genes are transcriptionally active and expressed (Nagalakshmi et al., 2008; Wilhelm et al., 2008; Wang et al., 2009). The detailed lists of genes for the RNA-Seq sequences identified in the cellobiose- and PYP-grown cells are presented in Supplementary Data Sheet 1. Each of the RPKM values of cellobiose-grown cells and RPKM of PYP-grown cells was the average of two replicates.
Our data from Solexa-read transcriptome measurement of PYP- vs. cellobiose-grown C. thermocellum illustrate some key characteristics of the results. First, the obtained RPKM values for most of the transcripts of the active protein-coding genes in PYP- and cellobiose-grown C. thermocellum, are in the range of $1-50,000$ (which can be revealed by re-sorting the Supplementary Data Sheet 1). Such range of RPKM values is comparable to the reported range of RPKM values in RNA-Seq whole-transcriptome analysis of other organism samples (Tang et al., 2009).

The gene expression levels can be classified into four levels: low, moderate, high, and very high, as illustrated in the $\mathrm{X}$ axis in Figure 3. While the RNA-Seq data sets of PYP- vs. cellobiosegrown cells had comparable numbers of genes that fall in the moderate expression levels (i.e., both $40 \%$ of the active proteinencoding genes), the PYP-grown cells had more highly expressed genes (41 vs. $33 \%$ in that of cellobiose-grown culture; Figure 3 ). In contrast, cellobiose-grown cells had more lowly expressed genes (26 vs. $18 \%$ in that PYP-grown culture). 


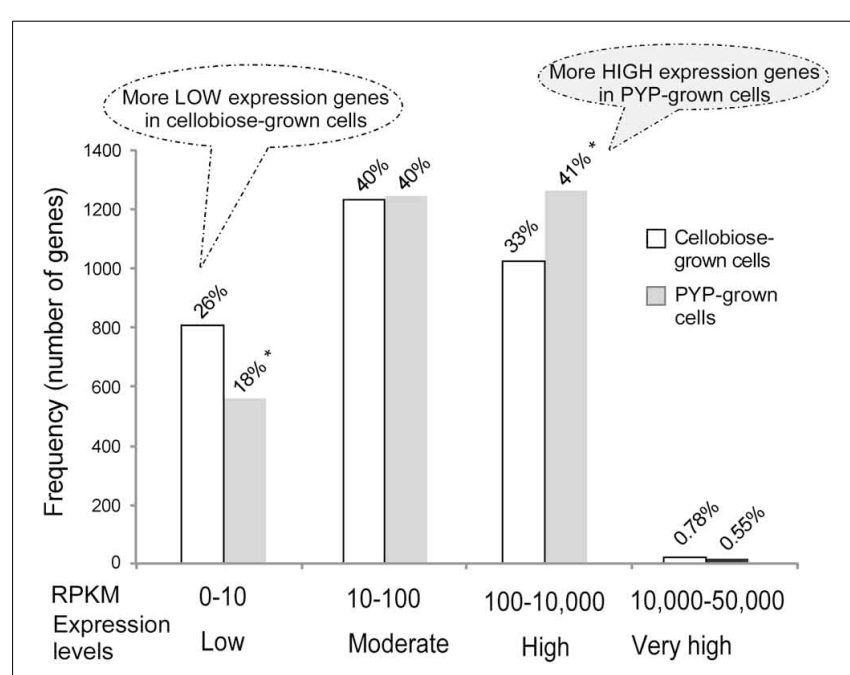

FIGURE 3 | RPKM frequency histogram of transcripts from RNA-Seq of pretreated yellow poplar (PYP)- vs. cellobiose-grown $C$. thermocellum cells. The diagram shows the distribution of the number of genes expressed at different RPKM levels. The total number of protein coding genes aligned with the RNA-Seq was 3081; the percentage value above each bar indicates the genes at specific expression level accounting for the proportion of total number of genes. The * mark indicates that significantly different frequencies (i.e., numbers of genes) were observed between the two RNA-Seq data sets from PYP-vs. cellobiose-grown C. thermocellum cells.

\section{CHANGES IN GLOBAL GENE EXPRESSION AND CLUSTERS OF ORTHOLOGOUS GROUPS (COG) ANALYSIS}

To compare the differential expression of genes between PYPand control cellobiose-grown cells, fold changes (FC) were computed as the ratio of the RPKM values obtained for individual genes in PYP- against cellobiose-grown cells. Analysis of changes in global gene expression had identified 1211 genes that show a two-fold or greater increase in expression (i.e., $F C \geq 2.0$ ) or detected in PYP-grown cells only (referred as PYPO genes), and 314 genes with a two-fold or greater decrease (i.e., $F C \leq 0.5$ ) in transcript abundance for PYP- against cellobiose-grown cells or detected in cellobiose-grown cells only (referred as CBO genes), as listed in Supplementary Data Sheet 2 with related statistic analyses showing that they were statistically significant.

The COG distribution for these up- and down-regulated genes in the transcriptome was determined and the result is shown in Figure 4. The top two categories for up- and down-regulated genes belong to the categories "general function prediction, [R]" (equivalent to unclassified) and "inorganic ion transport and metabolism, $[\mathrm{P}]$." A closer examination of the distribution chart reveals that for the up-regulated genes, the categories "signal transduction mechanisms, [T]," "amino acid transport and metabolism, [E]," and "energy production and conversion, [C]" (which is important for the energy-consuming process for biofuel end-product production), were also the most well-represented categories in the transcriptome, with the number of induced genes above 200 for each category (Figure 4).

An important category of COG for the degradation and utilization of lignocellulosic substrate is "carbohydrate transport and metabolism, [G]," which includes primarily cellulosome-related genes. In this category, the number of up- and down-regulated genes is 175 and 46, respectively (Figure 4). These differentially expressed genes are described in detail in later section.

\section{VALIDATION OF RNA-Seq RESULTS WITH QUANTITATIVE REVERSE TRANSCRIPTION-PCR}

Quantitative reverse transcription-PCR (qRT-PCR) is a well accepted method for verifying microarray (Ferreira et al., 2010; Yang et al., 2012) and RNA-Seq data (Cusick et al., 2012; Huang et al., 2012; Ji et al., 2013). We used this method to validate the expression patterns of 12 selected genes in independent biological replicates. The selected genes mainly represented different functional categories involving in cellulosome, hydrogen and ethanol production and had a range of FC values based on RNA-Seq. In addition to these 12 genes, the gene RecA was chosen as a reference gene to normalize the real time RT-PCR data because it has been commonly used as reference gene for $C$. thermocellum (Stevenson and Weimer, 2005). As a further confirmation for the use of this gene as reference gene, RecA did not differ in expression in our cell samples grown on two substrates, the values being RPKM 1166 for PYP-grown cells vs. RPKM 1237 for cellobiose-grown cells. The primers for all 13 genes, along with one-by-one comparisons of the fold-changes in expression of each gene as measured by RNA-Seq and qRT-PCR, are listed in Supplementary Data Sheet 3, section 1. Most of the qRT-PCR data matched the RNA-Seq based FC values with a correlation coefficient of 0.95 for the set of 12 selected genes, which indicated that our RNA-Seq result is accurate and the conclusion from RNA-Seq should be reliable.

\section{TRANSCRIPTIONAL CHANGES OF CELLULOSOMAL COMPONENTS}

Cellulosome is an extracellular supramolecular machine that can efficiently degrade crystalline cellulosic substrates and associated plant cell wall materials. We are especially interested in the genes encoding cellulosomal component proteins in response to cellulosic substrates, which can lead to the identification of regulatory or rate-limiting components regarding cellulolysis.

The number of reported cellulosomal genes in the genome of ATCC 27405 strain has been increasing from the initial numbers of 71 (Zverlov et al., 2005) and 72 (Zverlov and Schwarz, 2006), to more recently, 81 genes (Raman et al., 2011). Note that the latter number is consistent with the updated genome annotation. Of these 81 genes, all were detected transcriptionally in this study. The list of 81 cellulosomal genes with their FC values is described in Table 2.

Analysis of this sub-transcriptome showed the following features: first, the overall cellulosome-associated genes were upregulated significantly in PYP- vs. cellobiose-grown cells, reflected by the facts that out of the 81 cellulosomal genes, 47 (i.e., $58 \%$ ) were up-regulated with $F C \geq 2.0$. In contrast, only 4 out of 81 (i.e., about 5\%) cellulosomal genes, including a GH5 gene (Cthe_2193), CelU (Cthe_2360), XghA (Cthe_1398), and Cthe_0438, were down-regulated by two-fold or detected in celllobiose-grown cells only. The overall average FC value for all these 81 cellulosomal genes is 3.5 (see the bottom row in Table 2), 


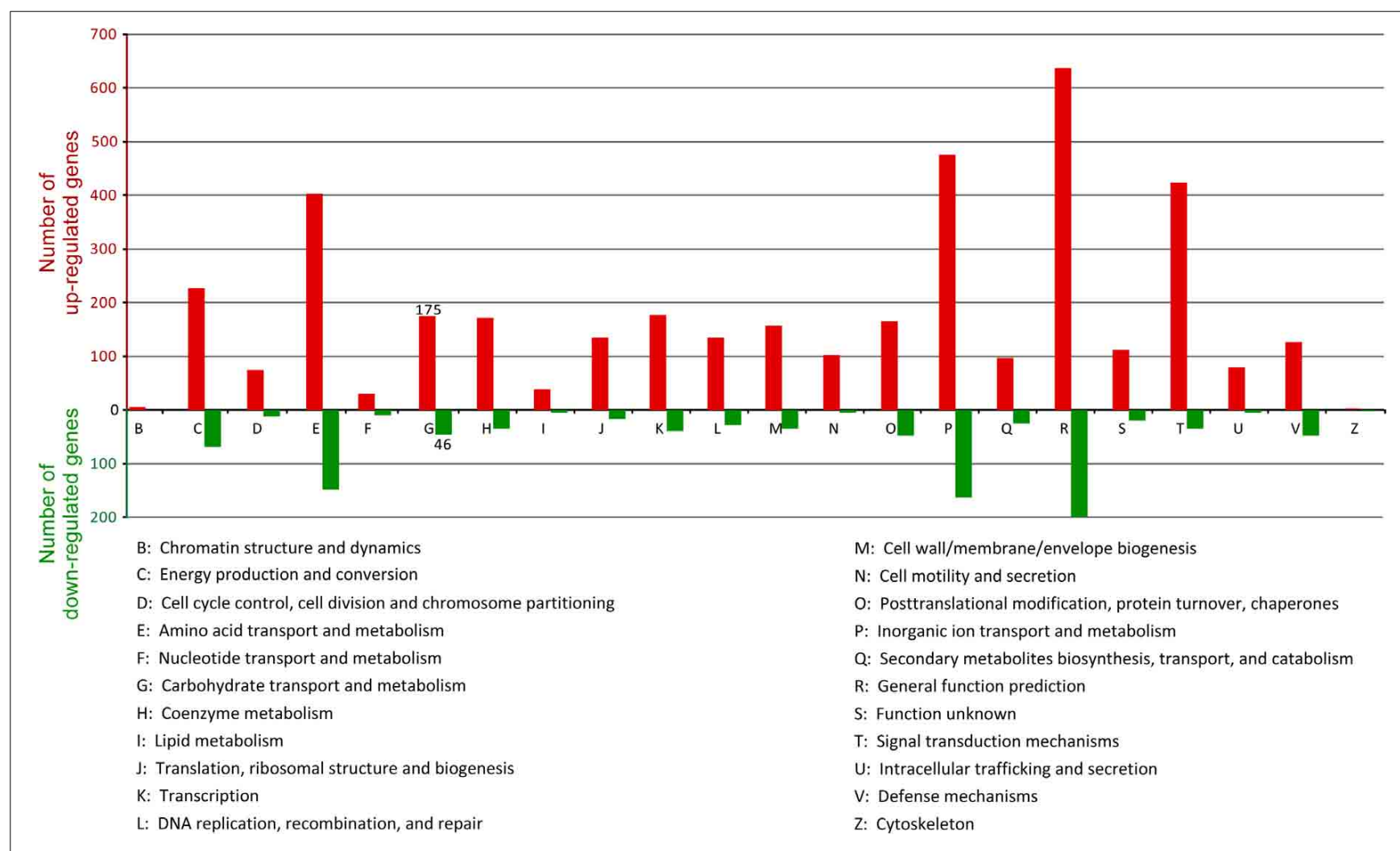

FIGURE 4 | COG functional categories of the 1211 up- and 314 down-regulated genes in PYP-grown cells compared with cellobiose-grown cells. Note that since COG annotation classes overlap, the sum of COG annotated genes is larger than the number of total up- and down-regulated genes analyzed.

suggesting the whole cellulosome machinery was "geared up" at the late stationary phase on PYP substrate.

Secondly, the primary scaffoldin, CipA, and the main secondary scaffoldins, OlpB, Orf2P, and SdbA, have shown significant up-regulation and were found to have the highest abundance on the transcriptional level. This implies that the cellulosomal system is crucial for the efficient degradation of pretreated PYP. Our data further verifies the notion that the C. thermocellum cellulosome is the main contributor to the extremely high activity observed in cellulose degradation. Additionally, one putative scaffoldin gene, Cthe_0736 (cellulosome anchoring protein) has been up-regulated as much as 2.8 times, i.e., FC 2.8 (Table 2, row 31 ). OlpC, which has been recently identified as an important outer layer protein - cellulosome anchoring protein cohesin subunit (Pinheiro et al., 2009), was also found to be up-regulated in this study (Cthe_0452, FC 4.1; Table 2, row 19).

Thirdly, the major cellulosomal cellulases, such as CelS (Cthe_2089, exo-, FC 4.5; RPKM 3848— the most abundant cellulosomal transcripts in PYP-grown cells), CelA (Cthe_0269, endo-, FC 1.7, RPKM 1885-the third most abundant transcript in PYP-grown cells), CbhA (Cthe_0413, exo/endo, FC 7.5, RPKM 367), and Cel124A (Cthe_0435, endo-; FC 2.0; RPKM 401) were remarkably up-regulated, as shown in Table 2. Exo- and endorefer to the substrate site upon which the GHs act. While exocellulases remove one or more sugar units from the ends of polysaccharide chain, endoglucanases randomly hydrolyze the internal glycosidic bonds of polysaccharides. Among the above listed enzymes, CelS was reported to display high synergy with the endo-Cel124A (Brás et al., 2011). Our observation that the CelS had the highest RPKM value in PYP-grown cells is consistent with a report that a knockout mutant of this gene showed a $\sim 60 \%$ reduction in cell cellulolytic performance (Olson et al., 2010), and is also consistent with a most recent finding that CelS gene was found to be highly expressed in C. thermocellum cells grown on both pretreated switchgrass and cottonwood substrates (Wilson et al., 2013a). This study furthered such transcriptional analysis by showing that the transcript of CelS is not only highly abundant, but also significantly responsive to biomass substrate, as its RPKM level in cellobiose-grown cell was 4.5 times lower (Table 2).

In addition, there was one cellulosome dockerin type I gene (Cthe_0438) for which the transcript was detected only in cellobiose-grown cells, with a low RPKM value of 4 (Table 2, row 81). This observation is consistent with a previous report regarding its absence in the sub-proteome of cellulosomes from cells grown on pretreated switchgrass (Raman et al., 2009). The domain architecture of Cthe_0438 is "DUF843-type I dockerin" (DUF indicates domain of unknown function). It is difficult to classify the association of this gene with any of the specific catalytic enzyme types, cellulases or hemicellulases, and thus this issue may warrant further studies. 
Table 2 | List of 81 cellulosomal genes with their RPKM and fold change (FC) values determined in PYP- vs. cellobiose-grown C. thermocellum cells.

\begin{tabular}{|c|c|c|c|c|c|c|}
\hline Row no. & Gene ID & Protein & GH, CBM family & RPKM of PYP cells & RPKM of cellobiose cells & $F C$ \\
\hline 1 & Cthe_0798 & Acetyl xylan esterase & CE3 & 483 & 20 & 24.3 \\
\hline 3 & Cthe_0912 & XynY & GH10, CE1, CBM22 & 261 & 19 & 13.8 \\
\hline 4 & Cthe_1890 & Dockerin type I cellulosome prot & $\mathrm{n} / \mathrm{a}$ & 45 & 3 & 14.0 \\
\hline 7 & Cthe_0412 & CelK & $\mathrm{GH} 9, \mathrm{CBM} 4$ & 1618 & 202 & 8.0 \\
\hline 8 & Cthe_0413 & CbhA & GH9, CBM4 & 367 & 49 & 7.5 \\
\hline 9 & Cthe_0660 & $\beta$-1,3-glucanase & GH81 & 4 & 1 & 6.7 \\
\hline 10 & Cthe_3077 & CipA & CBM3 & 2135 & 363 & 5.9 \\
\hline 11 & Cthe_3078 & OlpB & n/a & 333 & 59 & 5.6 \\
\hline 16 & Cthe_2089 & CelS & $\mathrm{GH} 48$ & 3848 & 864 & 4.5 \\
\hline 17 & Cthe_0043 & CelN & $\mathrm{GH} 9, \mathrm{CBM} 3$ & 122 & 28 & 4.4 \\
\hline 18 & Cthe_2147 & CelO & GH5, CBM3 & 63 & 15 & 4.3 \\
\hline 19 & Cthe_0452 & $\mathrm{OlpC}$ & $\mathrm{n} / \mathrm{a}$ & 140 & 34 & 4.1 \\
\hline 20 & Cthe_0661 & Ricin B lectin & GH43, CBM13 & 62 & 17 & 3.8 \\
\hline 21 & Cthe_2549 & Dockerin type I cellulosome prot & $\mathrm{n} / \mathrm{a}$ & 27 & 7 & 3.7 \\
\hline 22 & Cthe_2811 & ManA & GH26, CBM & 517 & 153 & 3.4 \\
\hline 23 & Cthe_1806 & Cellulosome prot, dockerin type I & Dockerin type I & 95 & 29 & 3.3 \\
\hline 24 & Cthe_0405 & CelL & $\mathrm{GH} 5$ & 96 & 29 & 3.3 \\
\hline 25 & Cthe_0246 & PL11 & PL11, CBM6 & 119 & 36 & 3.3 \\
\hline 33 & Cthe_0640 & Cellulosome prot, dockerin type I & $\mathrm{n} / \mathrm{a}$ & 50 & 18 & 2.7 \\
\hline 34 & Cthe_2761 & GH9 & GH9, CBM3 & 44 & 16 & 2.7 \\
\hline 35 & Cthe_0032 & $\mathrm{GH} 26$ & $\mathrm{GH} 26$ & 423 & 159 & 2.7 \\
\hline 36 & Cthe_2195 & CBM6 & CBM6 & 4 & 2 & 2.5 \\
\hline 37 & Cthe_2138 & $\mathrm{GH} 43$ & GH43, CBM42 & 13 & 5 & 2.4 \\
\hline 38 & Cthe_0258 & Cellulosome prot, dockerin type I & $\mathrm{n} / \mathrm{a}$ & 73 & 31 & 2.3 \\
\hline 39 & Cthe_2196 & $\mathrm{GH} 43$ & GH43, CBM6 & 2 & 1 & 2.3 \\
\hline 40 & Cthe_3012 & $\mathrm{GH} 30$ & GH30, CBM6 & 106 & 47 & 2.3 \\
\hline 41 & Cthe_2139 & $\mathrm{GH} 30, \mathrm{GH} 43, \mathrm{GH} 54$ & $\mathrm{GH} 30, \mathrm{GH} 43, \mathrm{GH} 54$ & 13 & 6 & 2.3 \\
\hline 42 & Cthe_2590 & XynD & GH10, CBM22 & 148 & 67 & 2.2 \\
\hline 43 & Cthe_0015 & Alpha-L-arabinofuranosidase B & GH43, GH54 & 203 & 92 & 2.2 \\
\hline 44 & Cthe_2038 & Cellulosome prot, dockerin type I & $\mathrm{n} / \mathrm{a}$ & 48 & 22 & 2.1 \\
\hline 45 & Cthe_1307 & SdbA & $\mathrm{n} / \mathrm{a}$ & 207 & 101 & 2.1 \\
\hline 46 & Cthe_2197 & $\mathrm{GH} 2$ & $\mathrm{GH} 2, \mathrm{CBM} 6$ & 2 & 1 & 2.0 \\
\hline 47 & Cthe_0435 & Cel124A & GH124 & 401 & 205 & 2.0 \\
\hline \multicolumn{7}{|c|}{30 GENES THAT DEFINED AS UNCHANGED $(2>$ FC $>0.5)$} \\
\hline 48 & Cthe_1838 & XynC & GH10, CBM22 & 746 & 405 & 1.8 \\
\hline 49 & Cthe_0191 & Proteinase inhibitor 14 , serpin & $\mathrm{n} / \mathrm{a}$ & 16 & 9 & 1.8 \\
\hline 50 & Cthe_2194 & CE1 & CE1, CBM6 & 12 & 7 & 1.8 \\
\hline
\end{tabular}


Table 2 | Continued

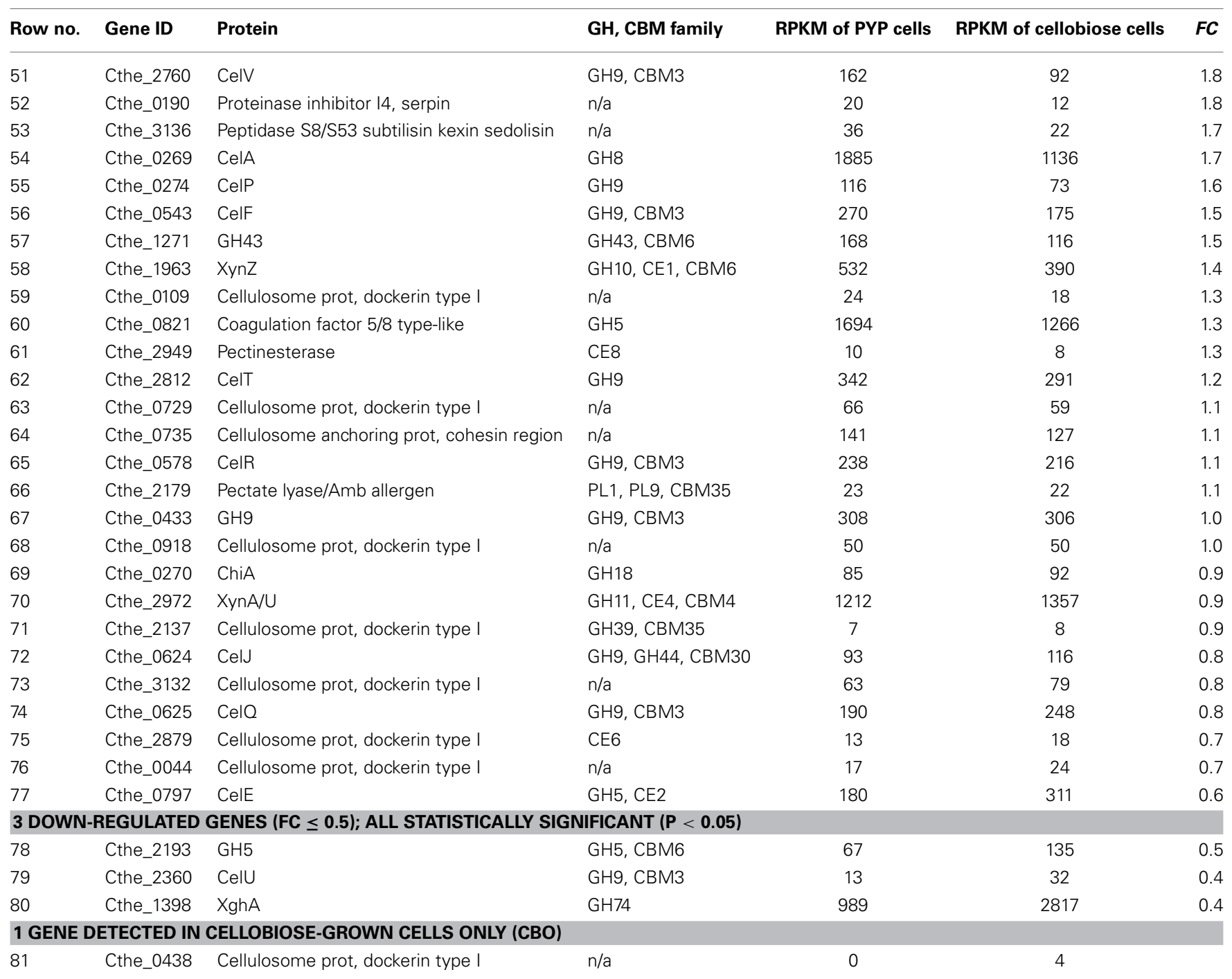

Average FC value for all genes (except the CBO)

The table is sorted in the order of $F C$ values. The glycoside hydrolase (GH) and carbohydrate-binding module (CBM) families were determined according to the annotations in GenBank and the CAZY database (www.CAZY.org). Other abbreviations: Cbh, cellobiohydrolase; CBO, transcript that detected only in cellobiosegrown cells; CE, carbohydrate esterases; Cel, cellulase; Chi, chitinase; CipA, cellulose-integrating protein A; Lic, lichinase; Man, mannanase; n/a, not applicable; OlpB, outer layer protein; ORF2p, open reading frame 2p; PL, polysaccharide lyase; Prot, protein; SdbA, scaffoldin-dockerin binding component $A ;$ XghA, xyloglucanase Xgh74A; Xyn, xylanase.

\section{EXPLORING THE CORRELATION BETWEEN RNA-Seq AND PUBLISHED PROTEOMIC DATA FOR CELLULOSOMAL GENES}

Literature reports have described the quantitative sub-proteomic data of cellulosomes extracted from cell-free culture filtrates of C. thermocellum grown to late stationary phase on cellobiose, Avicel, and other cellulosic substrates (Gold and Martin, 2007; Raman et al., 2009). In one study (Gold and Martin, 2007), an "emPAI," defined as the exponentially modified protein abundance index, showed a linear relationship with protein concentration and was normalized to the value obtained for CipA. Similarly, in another study (Raman et al., 2009), the normalized spectral abundance factor (NSAF) represented the number of spectral counts divided by the number of amino acid residues in the protein and was also normalized to the value obtained for CipA. To determine whether or not a correlative relationship exists between these published cellulosomal sub-proteomic data and the RNA-Seq RPKM data in the present study, we first retrieved the emPAI/CipA and NSAF/CipA data from the literature (Supplementary Data Sheet 3, section 2), and then plotted it against the $\log 2(\mathrm{RPKM})$ data for the genes encoding the same proteins in this study. The results showed that despite the fact that the plotted data were obtained from three different research groups, there remain strong correlations between the RNA-Seq RPKM and the protein abundance indicators of emPAI/CipA and 


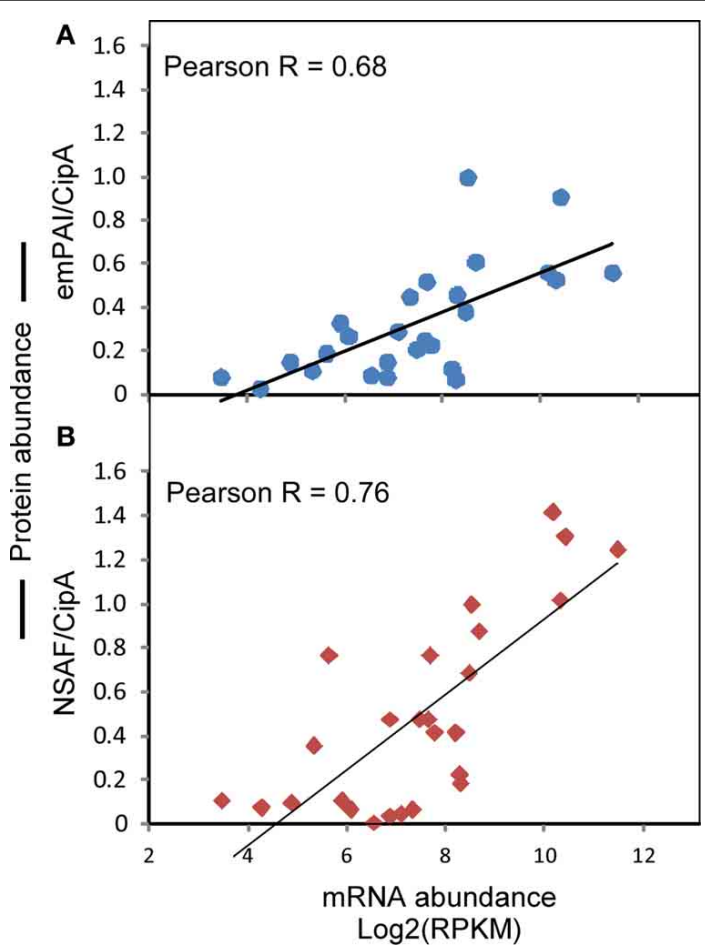

FIGURE 5 | Correlation between the observed gene abundances in this study and the published protein abundance data of cellulosomal components. (A) Correlation between Log2(RPKM) and emPAI/CipA. (B) Correlation between Log2(RPKM) and NSAF/CipA. The literature sources for data of emPAI/CipA and NSAF/CipA are described in the Results and Discussion section.

NSAF/CipA, with Pearson correlation coefficient values being 0.68 and 0.76 , respectively (Figures 5A,B).

\section{NON-CELLULOSOMAL GLYCOSIDE HYDROLASE PROTEINS AND "FREE" CELLULASES}

In addition to the GHs existing in cellulosomes, the $C$. thermocellum genome includes non-cellulosomal genes coding for GHs, among which 12 were up-regulated by more than twofold (Table 3). Notably, both CelC (Cthe_2807) and Lic16A (Cthe_2809), the two members in the putative CelC- GlyR3Lic16A operon (Newcomb et al., 2011), were up-regulated and predicted to be extracellular and/or bacterial cell wall associated (Table 3). This was consistent with a recent report that genes in this operon were mainly expressed in the stationary phase (very little during exponential phase) of pure cellulose (Avicel) fermentation (Raman et al., 2011). In addition, Lic16A contains CBM54 and a tandem of four CBM4s, in which both types of CBMs are able to bind xylan; as well as cellulose (Dvortsov et al., 2010). Remarkably, the grouping of four CBM4s in this protein has an $\sim 100$-fold higher binding constant for xylan and cellulose than that of a protein with a single CBM4 module (Dvortsov et al., 2012).

It is surprising that Cel9I (Cthe_0040), an important noncellulosomal processive endoglucanase that could digest crystalline cellulose with high efficiency, showed low abundance and FC value (RPKM 62 in PYP-grown cells, FC 1.3; see Supplementary Data Sheet 1, row no. 1875). Similarly, the only non-cellulosomal exo-cellulase CelY (Cthe_0071, exo-) also showed both low abundance and FC value (RPKM 61, FC 1.4, see Supplementary Data Sheet 1 , row no. 1741), which is consistent with a recent report that knocking out the CelY gene had no significant impact on the cellulolytic capacity of the strain (Olson et al., 2010). Based on the evidence above, it would seem that the free-enzyme system plays a less important role than does the cellulosome system in the degradation of PYP by C. thermocellum.

\section{RESPONSIVE SIGMA FACTORS COUPLED WITH MEMBRANE-ASSOCIATED ANTI-SIGMA FACTORS AS A MECHANISM FOR BIOSENSING BIOMASS SUBSTRATES}

Because COG analysis had revealed that the category of "signal transduction mechanisms, $[\mathrm{T}]$ " was among the most represented categories in the transcriptome of PYP-grown cells, we checked the genes related to the signal transmission attributed to sensing cellulose and other polysaccharide substrates. Recently, it was proposed the possible role of seven membrane-associated RsgI-like anti-sigma factors (referred to as RsgI) and their sigma factor partners (referred as SigI) in extracellular carbohydrate-sensing and glycosidase gene regulation in C. thermocellum (Kahel-Raifer et al., 2010; Nataf et al., 2010). In their proposed model, RsgI senses the presence of cellulose and other biomass components in the extracellular medium via its CBM domains, whereas SigI mediates the intracellular activation of different glycosidase genes (Kahel-Raifer et al., 2010; Nataf et al., 2010; Bahari et al., 2011). These predicted gene pairs include: SigI1- RsgI1 (Cthe_0058- Cthe_0059), oI2-RsgI2 (Cthe_0268- Cthe_0267), oI3-RsgI3 (Cthe_0315- Cthe_0316), бI4-RsgI4 (Cthe_0403- Cthe_0404), $\sigma$ I5-RsgI5 (Cthe_1272Cthe_1273), $\sigma$ I6-RsgI6 (Cthe_2120- Cthe_2119), and $\sigma 24 \mathrm{C}$ Rsi24C (Cthe_1470-Cthe_1471). Among them, three SigI genes (Cthe_0058, Cthe_0268, Cthe_0403) were up-regulated during later stages of pure cellulose (Avicel) fermentation (Raman et al., 2011).

In this study, while the transcripts of all seven pairs of genes had been detected (see Supplementary Data Sheet 1), three pairs of genes and an extra SigI-RsgI pair (which was not included in the initial literature prediction shown above) were up-regulated with FC values above 3.0 in the PYP- against cellobiose-grown cells at the late stationary phase, as described below:

(1) oI3-RsgI3 (Cthe_0315- Cthe_0316), with FC 4.2, and 5.9, respectively. According to literature, RsgI3 contains a PA14 dyad domain that target pectin (Nataf et al., 2010). The differential expression of this pair was not previously reported in cellulose-grown $C$. thermocellum.

(2) $\sigma$ I4-RsgI4 (Cthe_0403-Cthe_0404), with FC 3.1, and 3.9, respectively. RsgI4 contains CBM3 that binds cellulose. This observation supports previous observation of this pairs being involved in the time course of pure cellulose fermentation by C. thermocellum (Raman et al., 2011).

(3) $\sigma^{24 C}$-Rsi24C (Cthe_1470- Cthe_1470), with FC 4.0 and 5.9, respectively. Note that Rsi24C contains a module that resembles GH5 that target cellulose based on literature (Nataf et al., 
Table 3 | Twelve non-cellulosomal GH genes that were up-regulated by two or more folds in pretreated yellow poplar (PYP)- compared with cellobiose-grown $C$. thermocellum cells.

\begin{tabular}{llll}
\hline Gene ID & Protein and GH family & FC & Cellular location prediction $^{\mathbf{b}}$ \\
\hline Cthe_1256 & GH3; bglB; $\beta$-glucosidase B & 28.6 & n/a \\
Cthe_1428 & GH1; bgl & 9.1 & Cytoplasmic (Psortb) \\
Cthe_2744 & GH23; lytic transglycosylase & 7.5 & Membrane (UniProt) \\
Cthe_2809 & GH16, CBM4, CBM54; $\beta$-1,3-1,4-glucanase, Lic16A & 6.0 & Cell wall (Psortb) Extracellular (Psortb) \\
Cthe_1471 & GH5; Rsi24C & 5.9 & Membrane (Bahari et al., 2011) \\
Cthe_2807 & GH5; CelC, endoglucanase C & 5.0 & Extracellular (Psortb) \\
Cthe_0043 & GH9, CBM3; CelN, Cel9N & 4.4 & Membrane (Psortb) \\
Cthe_0322 & GH3 & 4.3 & Extracellular (Uniprot) \\
Cthe_2191 & $\alpha-g l u c a n$ branching enzyme; GH13 & 3.3 & Membrane (Burton, 2010) \\
Cthe_2548 & GH51, $\alpha-N$-arabinofuranosidase & 3.3 & Cytoplasmic (UniProt) \\
Cthe_1221 & GH94, GT84; glycosyltransferase & 3.0 & Membrane (Psortb) \\
Cthe_0795 & GH13, CBM34; $\alpha$-amylase, catalytic region & 2.2 & Cytoplasmic (Psortb) \\
\hline
\end{tabular}

${ }^{a}$ GH family characterization was based on CAZy database (http://www.cazy.org/).

${ }^{b}$ The cellular location was based on three sources: literature, UniProt database (http://www.uniprot.org/f, or prediction by Psortb program (http://www.psort.org/psortb/index.html). All listed genes were statistically significantly up-regulated $(p<0.05)$. n/a, not available.

2010). Similar to $\sigma \mathrm{I} 3$-RsgI3, the differential expression of this pair was also not previously reported in cellulose-grown C. thermocellum.

(4) Equally interesting, we found that another SigI-RsgI pair (Cthe_2521-Cthe_2522) was detected on transcriptome, with FC values of 3.9 and 4.8, respectively (Supplementary Data Sheet 1). The protein of sigma factor Cthe_2521 had been detected in the proteome of $C$. thermocellum cultured on cellobiose (Rydzak et al., 2012), but was not in the initial 7 pairs of SigI-RsgI proposed for polysaccharide signal transmission in the literature (Nataf et al., 2010). The possible role of this pair warrants further investigation.

\section{GENES RELATED TO CELLODEXTRIN TRANSPORT AND PHOSPHORYLATION}

C. thermocellum has been reported to use ABC-type transporters for uptake of oligosaccharides derived from cellulose hydrolysis (Strobel et al., 1995), which is an important energyconserving mechanism by which importing long cellodextrins can reduce the cost of transport as one-ATP molecule is consumed per transport event. So far, four cellodextrin ABC transporters (carbohydrate-binding protein $\mathrm{CbpA}, \mathrm{B}, \mathrm{C}$, and $\mathrm{D}$ ) have been characterized for their substrate binding features (Nataf et al., 2009). Whereas CbpA (Cthe_0393) binds only to cellotriose (G3), CbpB (Cthe_1020) binds to G2-G5 cellodextrins, and CbpC (Cthe_2128) and D (Cthe_2446) bind to G3-G5 cellodextrins (Nataf et al., 2009; Rydzak et al., 2012). Several transcripts of these annotated genes have been detected in the transcriptome of C. thermocellum (Raman et al., 2011; Riederer et al., 2011). Previously, six cellodextrin ABC transporter genes (Cthe_1862, Cthe_0391-0393, and 1019-1020, including CbpA and $\mathrm{CbpB}$ ) were found to be expressed at high levels throughout the course of Avicel alone fermentation (Raman et al., 2011). Most recently, Cthe_0391-0393 were found to be highly expressed on both pretreated switchgrass and cottonwood substrates (Wilson et al., 2013a). This study furthered such transcriptional analysis by comparing the gene expression between PYP- and cellobiose-grown cells at late stationary phase. A total of 12 transcripts of cellodextrin ABC transporters have been detected (Supplementary Data Sheet 3, section 3; also Figure 6), among which, seven were up-regulated in PYP- against cellobiose-grown cells, with FC values in the range of 3.1-10.3, suggesting that the PYP-grown cells had a mechanism for enhancing the uptake and utilization of polysaccharides derived from biomass substrates.

For the subsequent phosphorolytic cleavage of the imported oligosaccharides and cellobiose, this study identified the transcripts of one cellodextrin phosphorylase (CDP, Cthe_2989) and two cellobiose phosphorylases (CEP, Cthe_0275 and Cthe_1221). Among these, the CEP Cthe_1221 was up-regulated with a FC value of 3.0 (Supplementary Data Sheet 3, section 3; also Figure 6), suggesting this gene may warrant further studies.

\section{GENES RELATED TO GLYCOLYSIS, PYRUVATE CATABOLISM, AND END-PRODUCT SYNTHESIS}

The deduced pathway for cellulolysis, glycolysis, ethanol, and $\mathrm{H}_{2}$ production in $C$. thermocellum ATCC 27405 is illustrated in Figure 6, which is in accordance to accumulated literature findings (Demain et al., 2005; Rydzak et al., 2009, 2011; Riederer et al., 2011; Carere, 2013). The set of gene IDs for the enzymes involved in above pathway were retrieved from the KEGG PATHWAY database (http://www. genome.jp/kegg) (Kanehisa et al., 2008), and were shown in Supplementary Data Sheet 3, section 3 with their FC values in PYP- vs. cellobiose-grown cells. Out of the listed genes, 18 and 12 were significantly up- and down-regulated, as shown in red and green text in Supplementary Data Sheet 3, section 3, respectively.

For pyruvate catabolism and end-product synthesis, $C$. thermocellum may convert pyruvate into (1) formate via pyruvate formate lyase (PFL) and pyruvate formate lyase activating enzyme (PFL-AE), (2) lactate via lactate dehydrogenase (LDH), (3) $\mathrm{CO}_{2}$, 


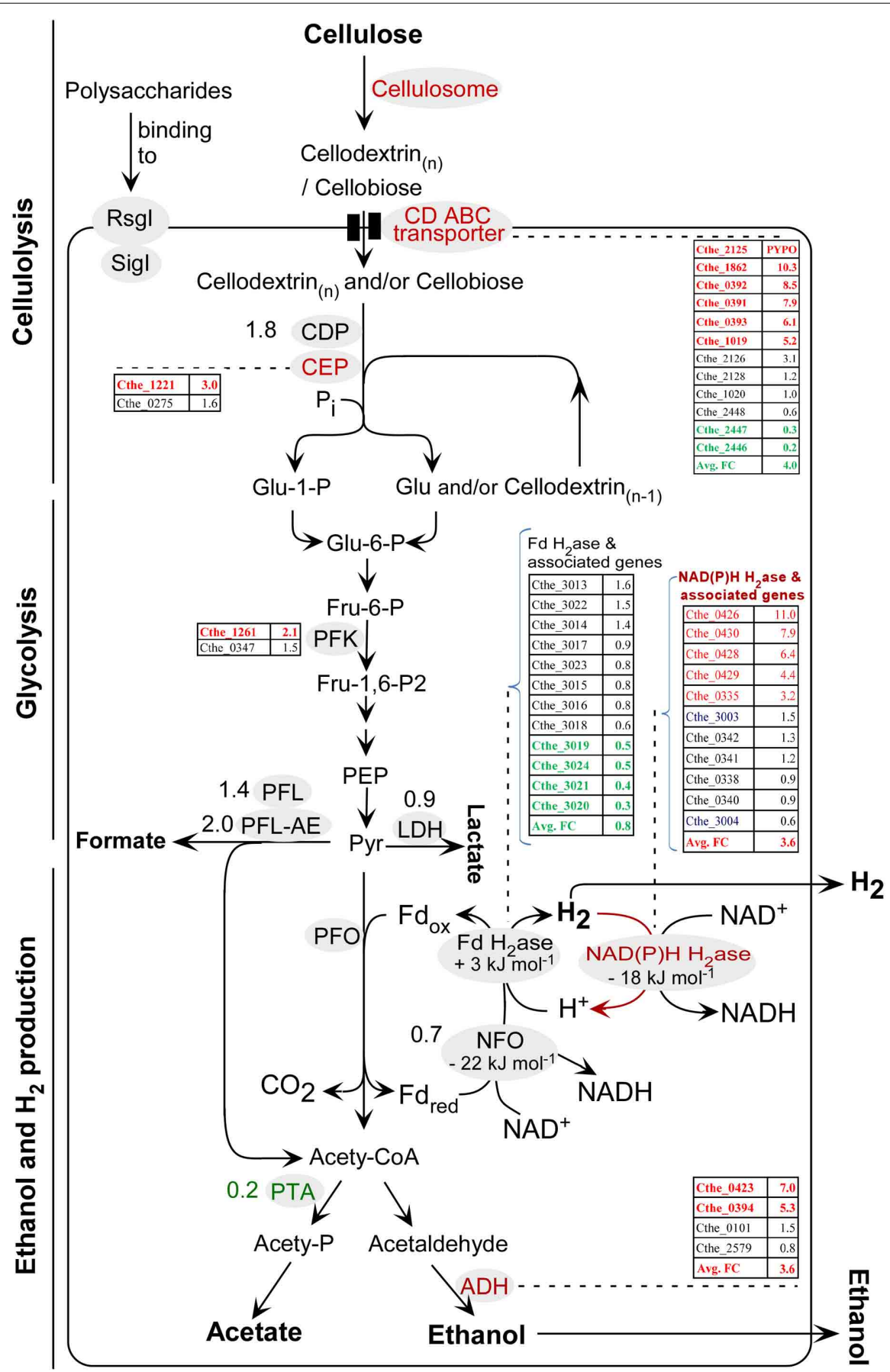

FIGURE 6 | Diagram for the primary steps in the conversion of cellulose to fermentation products using $\boldsymbol{C}$. thermocellum ATCC 27405. Sigl-Rsgl (sigma factors coupled with membrane-associated Rsgl-like anti-sigma factors) are also illustrated in this diagram as they have been proposed in literature for polysaccharide triggered signal transmission in regulation of $\mathrm{GH}$ family genes. Detailed nomenclature of enzymes and their associated gene IDs can be found in Supplementary Data Sheet 3, section 3 and Table 4 For enzymes associated with a single gene ID, the gene ID is not included in the figure; instead, its fold change (FC) value is indicated directly beside the enzyme. For enzymes associated with multiple gene IDs, the gene IDs and their $\mathrm{FC}$ values are illustrated in sorted lists in the diagram, in order of either their FC values or locus IDs. Text in red and green represents the up-regulated and down-regulated genes, respectively, in PYP-against cellobiose-grown cells; in contrast, text in black represents the genes with no significant transcriptional changes between the two types of cells, i.e., $2.0>$ $F C$ value $>0.5$. Nomenclature of metabolites: CoA, coenzyme $A ; E C H$, energy conserving hydrogenase; Fd, ferredoxin; Fru, fructose; Glu, glucose; ox, oxidized; P, phosphate; PEP, phosphoenolpyruvate; Pi, inorganic phosphate; PYPO, transcript that was detected in PYP-grown cells only; Pyr, pyruvate; red, reduced. 
Table 4 | C. thermocellum genes encoding putative hydrogenases, sensory hydrogenases, and NADH:Fd oxidoreductases using ferredoxin and $N A D(P) H$ as electron carriers.

\begin{tabular}{lll}
\hline Locus ID Gene name & FC \\
\hline
\end{tabular}

\section{TYPE 1. [NiFe] $\mathrm{H}_{2}$ ase}

Fd-dependent ECH: Cthe_3013-Cthe_3024

Cthe $3013 \quad H y d r o g e n a s e$ expression/formation protein HypE

Cthe_3014 Hydrogenase formation HypD protein

Cthe_3015 Hydrogenase assembly chaperone hypC/HupF

Cthe_3016 [NiFe] hydrogenase maturation protein HypF

Cthe_3017 Hydrogenase accessory protein HypB

Cthe_3018 Hydrogenase expression/synthesis HypA

Cthe_3019 4Fe-4S ferredoxin, iron-sulfur binding

Cthe_3020 Ech hydrogenase subunit E (Fd H2 $\mathrm{H}_{2}$ ase)

Cthe_3021 Ech hydrogenase subunit D

Cthe_3022 Ech hydrogenase subunit C

Cthe_3023 Ech hydrogenase subunit B

Cthe_3024 Ech hydrogenase subunit A

TYPE 2. [FeFe] $\mathrm{H}_{2}$ ases

Type 2.1. Bifurcating: Cthe_0338-Cthe_0342; Cthe_0428-Cthe_0430

\begin{tabular}{lll}
\hline Cthe_0338 & NADH-dependent hydrogenases & 0.9 \\
Cthe_0340 & Ferredoxin & 0.9 \\
Cthe_0341 & NADH dehydrogenase (ubiquinone) & 1.2 \\
Cthe_0342 & [FeFe] H $\mathrm{H}_{2}$ ase (NADH H $\mathrm{H}_{2}$ ase) & 1.3 \\
Cthe_0428 & $\mathrm{NADH}$ dehydrogenase (ubiquinone) & 6.4 \\
Cthe_0429 & $\mathrm{NADH}$ dehydrogenase (ubiquinone) & 4.4 \\
Cthe_0430 & [FeFe] $\mathrm{H}_{2}$ ase & 7.9
\end{tabular}

Type 2.2. Sensory: Cthe_0425-Cthe_0426

\begin{tabular}{llr}
\hline Cthe_0425 & Putative hydrogenase & 9.9 \\
Cthe_0426 & [FeFe] hydrogenase & 11.0
\end{tabular}

Type 2.3. NAD(P)H dependent: Cthe_3003-3004

\begin{tabular}{lll}
\hline Cthe_3003 & [FeFe] $\mathrm{H}_{2}$ ase, putative ${ }^{\mathrm{a}}$ & 1.5 \\
Cthe_3004 & Ferredoxin & 0.6
\end{tabular}

\section{OTHER RELATED GENES}

Cthe_0335 Hydrogenase large subunit- like protein

3.2

In addition, hydrogenase maturation proteins are also listed. The table is arranged according to the hydrogenase classification described by two literature sources, as cited in the related Results and Discussion section; within each sub-category, it was arranged in order of locus IDs. For each gene, fold change (FC) value was calculated by dividing the RPKM of PYP cells by RPKM of cellobiose cells, as listed in Supplementary Data Sheet 1. RPKM, reads per kilobase of exon model per million mapped reads. Text in black represents the genes with no significant transcriptional changes between the two types of cells, i.e., $2.0>F C$ value $>0.5$. Text in red and green represents the genes that found to be significantly up-regulated and down-regulated, respectively, in PYP-against cellobiose-grown cells, based on statistic analysis in Supplementary Data Sheet 2. ECH, energy conserving hydrogenase. PYPO, transcript that detected in PYP-grown cells only.

${ }^{a}$ Cthe_3003 ([FeFe] $\mathrm{H}_{2}$ ase, putative) was characterized as Fd-linked by Schut and Adams (2009), but as NAD(P)H dependent recently by Carere et al. (2012).
$\mathrm{Fd}_{\text {red }}$, and acetyl-CoA, in which acetyl-CoA eventually leads to the production of acetate and ethanol, the $\mathrm{Fd}_{\text {red }}$ leads to the formation of hydrogen (Figure 6). The up-regulation of two out of four alcohol dehydrogenase $(\mathrm{ADH})$ genes, namely Cthe_0423 (FC value 7.0) and Cthe_0394 (FC value 5.3), raise the average $\mathrm{FC}$ value for $\mathrm{ADH}$ s to 3.6. In contrast, the phosphotransacetylase (PTA; Cthe_1029), whose activity had been verified in C. thermocellum (Lamed and Zeikus, 1980), was significantly down-regulated in PYP- against cellobiose-grown cells, with an FC value of 0.2. These data suggest that based on mRNA profiling, carbon flux of acetyl-CoA may preferentially be channeled to ethanol production in PYP-grown cells. The observed decrease of acetate/ethanol ratio, from 1.08 in cellobiose-grown cells to 0.92 in PYP-grown cells (Table 1), supports this carbon shift at the late stationary phase of PYP-grown cells.

Lactate and formate were not monitored in this study as previous study has shown that in the late growth phase of $C$. thermocellum cultures grown on cellobiose substrate, lactate, and formate together represent only a small fraction of the total end products produced (Islam et al., 2006). Future studies should also monitor the production of lactate, formate and $\mathrm{CO}_{2}$, which could provide a whole picture for carbon balance for the cells utilizing biomass substrates.

\section{DYNAMICS FOR $\mathrm{H}_{2}$ PRODUCTION WITH PYP AS SUBSTRATE}

C. thermocellum genes encoding putative hydrogenases and sensory hydrogenases using ferredoxin and $\mathrm{NAD}(\mathrm{P}) \mathrm{H}$ as electron carriers are listed Table 4. In addition, hydrogenase maturation proteins are also listed. The classification of the above hydrogenases are mainly based on literatures that systematically characterized the putative hydrogenases in C. thermocellum and other species (Schut and Adams, 2009; Carere et al., 2012). Briefly, there are two types of hydrogenases $\left(\mathrm{H}_{2}\right.$ ases $)$ according to the metal content in the respective active sites:

The first type is [NiFe] $\mathrm{H}_{2}$ ase, a putative $\mathrm{Fd}$-dependent energy converting hydrogenase $(\mathrm{ECH})$. Its hexameric structural subunits are encoded by Cthe_3019-Cthe_3024, with assembly of its active site assisted by a suite of maturation proteins encoded by Cthe_3013-Cthe_3018 (Table 4, Figure 6). This study showed that the FC values for 7 of the 12 detected Fd- $\mathrm{H}_{2}$ ase and related genes (Cthe_3013-Cthe_3015, Cthe_3017Cthe_3018, and Cthe_3022-Cthe_3023) were in the range of 0.6-1.6, suggesting an unchanged status at the transcriptional level for these genes. However, the remaining five genes (Cthe_3019-Cthe_3021, Cthe_3023-Cthe_3024) encoding the $\mathrm{Fd}-\mathrm{H}_{2}$ ase structural subunits were significantly down-regulated with FC values between 0.3 and 0.5 , which might account for the observed lower $\mathrm{H}_{2}$ yield in PYP-grown cells, compared to the control cellobiose-grown cells. This finding implies that the [NiFe] $\mathrm{ECH} \mathrm{H}_{2}$ ase likely functions in the $\mathrm{H}_{2}$ production direction since its down-regulation led to less $\mathrm{H}_{2}$ accumulation in the culture headspace.

The second type is [FeFe] $\mathrm{H}_{2}$ ases that include two bifurcating $\mathrm{H}_{2}$ ases: both being trimeric, and encoded by Cthe_0338Cthe_0342 and Cthe_0428-Cthe_0430, respectively. Only seven genes are listed in Table 4 because Cthe_0339 is annotated as 
histidine kinase in GenBank protein database. The second type also includes a sensory $\mathrm{H}_{2}$ ase (Cthe_0425-Cthe_0426) and a $\mathrm{NAD}(\mathrm{P}) \mathrm{H}$ dependent $\mathrm{H}_{2}$ ase (Cthe_3003-Cthe_3004), as listed in Table 4. The data showed that the FC values for the trimeric bifurcating $\mathrm{H}_{2}$ ase genes (Cthe_0338, Cthe_0340-Cthe_0342) and the $\mathrm{NAD}(\mathrm{P}) \mathrm{H}$ dependent $\mathrm{H}_{2}$ ase genes (Cthe_3003-3004) were in the range of $0.6-1.5$, indicating an unchanged status for their transcription (Figure 6). A homolog of this trimeric $\mathrm{H}_{2}$ ase has been uncovered in Acetobacterium woodii which functions in $\mathrm{H}_{2}$ consumption yielding reduced $\mathrm{Fd}$ and $\mathrm{NAD}(\mathrm{P}) \mathrm{H}$ (Schut and Adams, 2009; Hess et al., 2013a,b). In contrast, genes encoding the trimeric bifurcating $\mathrm{H}_{2}$ ase (Cthe_0428-Cthe_0430) were significantly up-regulated in PYP-grown cells, with FC values of 6.4-7.9 (Figure 6). Based on literature (Schut and Adams, 2009; Calusinska et al., 2010), the trimeric [FeFe] $\mathrm{H}_{2}$ ase identified in $C$. thermocellum is a putative bifurcating hydrogenase. This cytoplasmic enzyme was initially characterized in Thermotoga maritima and uses both reduced ferredoxin and $\mathrm{NAD}(\mathrm{P}) \mathrm{H}$ as substrates, and functions in $\mathrm{H}_{2}$ production (Schut and Adams, 2009; Hess et al., 2013a). Yet the up-regulation of Cthe_0428Cthe_0430 (this work) led to decreased $\mathrm{H}_{2}$ production when cultured in PYP- vs. cellobiose-grown cells. As such, the exact role of the $C$. thermocellum trimeric $\mathrm{H}_{2}$ ase is unknown, which makes it hard to link the above transcriptional data to the observed lower $\mathrm{H}_{2}$ yield in PYP-grown cells. Further studies are warranted to investigate the exact direction (production or consumption of $\mathrm{H}_{2}$ ) for each of the above [FeFe] $\mathrm{H}_{2}$ ases.

In addition, the sensory $\mathrm{H}_{2}$ ase encoded by Cthe_0425Cthe_0426 had a FC value of 9.9 and 11.0, respectively (Table 4), and future studies are also needed to explore the implication of the significant up-regulation of these two genes in PYP-grown cells.

\section{GENES FOR DEGRADING HEMICELLULOSE AND LIGNIN IN FEEDSTOCK BIOMASS}

We noticed that some important hemicellulase genes, such as XynY (Cthe_0912, FC 13.8), XynD (Cthe_2590, FC 2.2), XynZ (Cthe_1963, FC 1.4), ManA (Cthe_2811, FC 3.4) were upregulated in PYP- against cellobiose-grown cells (Table 2; cellulosomal proteins). In addition, some auxiliary enzymes such as $\alpha$-N-arabinofuranosidase (Cthe_2548, FC 3.3; Table 3, noncellulosomal protein) were also up-regulated. This is probably caused by the adaptation of the strain to digest the PYP, of which the chemical composition and structure are much different from those of cellobiose. In contrast, we did not identify the primary genes involved in lignin modification and degradation, such as laccases, lignin peroxidases, or manganese peroxidases.

\section{GROWTH PHASE AND THE TRANSCRIPTOME OF CELLS}

The main focus of this study was to investigate the effects of different carbon sources (PYP vs. cellobiose) on the transcriptome of the cells. To attain this goal, the selection of sampling point was crucial. The experimental design of this study in choosing the late stationary phase is in accordance with literature (Gold and Martin, 2007; Raman et al., 2009). One merit for choosing this sampling point is that our transcriptomic data can be cross-analyzed with the literature's protein data of cellulosomes extracted from cellobiose-grown cells, which are used as one means of validating the transcriptomic data in this study.

Another merit in choosing this sampling point is that compared with other early growth stages (exponential or early stationary phases), the levels of simple, soluble sugars in PYP- and cellobiose-grown culture are both very low: due to either the limit of bacterial enzymes in releasing sugars from PYP or the depletion of cellobiose, respectively. In this sense, late stationary phase is relatively more suitable phase than other phases for comparing transcriptomes derived from different carbon substrate-grown cells in this study.

Nevertheless, it is noteworthy that different growth phases may cause some shifts to some metabolic pathways; thus future studies comparing cells grown on cellobiose vs. cells grown on woody biomass at the exponential phase would provide another angle to investigate the effects of biomass substrate on the transcriptomes of $C$. thermocellum cells, which may lead to the identification of both growth phase- and biomass substrate-responsive genes. In addition, the transcriptional profiles of these two cell samples could also be affected by the distinct concentrations of carbohydrate sources as well as the residual concentrations of metabolites/nutrients present at time of sampling, which will remain a challenge for future studies in managing these variables-some of which are nearly uncontrollable.

\section{CONCLUSIONS}

We conducted a RNA-Seq analysis of the transcriptional profiles of C. thermocellum grown on PYP and cellobiose, which is different from previous transcriptional studies that focused on degrading cellulose alone substrates, or sub-proteomic studies that focused on cellulosomal protein components. We found that nearly $60 \%$ of the genes encoding the protein components of the cellulosomes - the core machinery for cellulose degradationwere up-regulated, whereas only $5 \%$ were down-regulated. The top up-regulated cellulosomal genes, along with the responsive SigI-RsgI and cellodextrin transporter genes, present promising candidate genes for engineering $C$. thermocellum strains to improve their capacity in effectively converting lignocellulosic biomass substrates. Furthermore, the identified differentially expressed $\mathrm{NAD}(\mathrm{P}) \mathrm{H} \mathrm{H}_{2}$ ase, $\mathrm{ADH}$, and PTA genes may provide insight into how the cells regulate the production of $\mathrm{H}_{2}$ and ethanol under the carbon-limited condition.

\section{ACKNOWLEDGMENTS}

This work was funded by the Laboratory Directed Research and Development (LDRD) program at the National Renewable Energy Laboratory (NREL), and by the U.S. Department of Energy's Office of Science through the BioEnergy Science Center (BESC), Bioenergy Technology Office (DOE-BETO) and the Fuel Cell Technologies Office. This work was supported by DOE under Contract No. DE-AC36-08-GO28308 with NREL. Andrew Bowersox and Igor Bogorad were sponsored by DOE Academies Creating Teacher Scientists (ACTS) and Science Undergraduate Laboratory Internship (SULI) programs, respectively. We thank Dr. Katherine Chou of NREL for discussion. 


\section{SUPPLEMENTARY MATERIAL}

The Supplementary Material for this article can be found online at: http://www.frontiersin.org/journal/10.3389/fmicb.2014. 00142/abstract

Supplementary Data Sheet 1 | List of 3081 genes with their RPKM and fold change values determined in PYP- vs. cellobiose-grown $C$. thermocellum cells.

Supplementary Data Sheet 2 | List of 1211 up-regulated and 314 down-regulated genes in PYP-grown $C$. thermocellum cells.

Supplementary Data Sheet 3 | Section 1. Forward (F) and reverse (R) primer sequences for quantitative reverse transcription-PCR (qRT-PCR) analysis of selected genes in C. thermocellum. Section 2. RPKM values of cellulosomal genes based on this study and their protein abundance data retrieved from literature for cellobiose-grown $C$. thermocellum cells at the late stationary phase. Section 3. Fold change values for genes related to cellodextrin transport and phosphorylation, glycolysis, pyruvate catabolism and end-product synthesis in $C$. thermocellum.

\section{REFERENCES}

Alper, H., and Stephanopoulos, G. (2009). Engineering for biofuels: exploiting innate microbial capacity or importing biosynthetic potential? Nat. Rev. Microbiol. 7, 715-723. doi: 10.1038/nrmicro2186

Bahari, L., Gilad, Y., Borovok, I., Kahel-Raifer, H., Dassa, B., Nataf, Y., et al. (2011). Glycoside hydrolases as components of putative carbohydrate biosensor proteins in Clostridium thermocellum. J. Ind. Microbiol. Biotechnol. 38, 825-832. doi: 10.1007/s10295-010-0848-9

Baker, J. O., Vinzant, T. B., Ehrman, C. I., Adney, W. S., and Himmel, M. E. (1997). Use of a new membrane-reactor saccharification assay to evaluate the performance of celluloses under simulated ssf conditions. Appl. Biochem. Biotechnol. 63, 585-595. doi: 10.1007/BF02920456

Bayer, E. A., Belaich, J. P., Shoham, Y., and Lamed, R. (2004). The cellulosomes: multienzyme machines for degradation of plant cell wall polysaccharides. Annu. Rev. Microbiol. 58, 521-554. doi: 10.1146/annurev.micro.57.030502. 091022

Bayer, E. A., Chanzy, H., Lamed, R., and Shoham, Y. (1998). Cellulose, cellulases and cellulosomes. Curr. Opin. Struct. Biol. 8, 548-557. doi: 10.1016/S0959440X (98)80143-7

Bayer, E. A., Lamed, R., and Himmel, M. E. (2007). The potential of cellulases and cellulosomes for cellulosic waste management. Curr. Opin. Biotechnol. 18, 237-245. doi: 10.1016/j.copbio.2007.04.004

Brás, J. L., Cartmell, A., Carvalho, A. L. M., Verzé, G., Bayer, E. A., Vazana, Y., et al. (2011). Structural insights into a unique cellulase fold and mechanism of cellulose hydrolysis. Proc. Natl. Acad. Sci. U.S.A. 108, 5237-5242. doi: 10.1073/pnas.1015006108

Brown, S. D., Lamed, R., Morag, E., Borovok, I., Shoham, Y., Klingeman, D. M., et al. (2012). Draft genome sequences for Clostridium thermocellum wildtype strain YS and derived cellulose adhesion-defective mutant strain AD2. J. Bacteriol. 194, 3290-3291. doi: 10.1128/JB.00473-12

Burton, E. (2010). Proteomic Analysis of Cellulose Metabolism in Clostridium thermocellum. Master thesis, Concordia University, Montreal.

Calusinska, M., Happe, T., Joris, B., and Wilmotte, A. (2010). The surprising diversity of clostridial hydrogenases: a comparative genomic perspective. Microbiology 156, 1575-1588. doi: 10.1099/mic.0.032771-0

Carere, C. R., Rydzak, T., Verbeke, T. J., Cicek, N., Levin, D. B., and Sparling, R. (2012). Linking genome content to biofuel production yields: a meta-analysis of major catabolic pathways among select $\mathrm{H} 2$ and ethanol-producing bacteria. BMC Microbiol. 12:295. doi: 10.1186/1471-2180-12-295

Carere, R. C. (2013). Genomics of Cellulolytic Clostridia and Development of Rational Metabolic Engineering Strategies. Ph.D., University of Manitoba.

Chen, Z., and Duan, X. (2011). Ribosomal RNA depletion for massively parallel bacterial RNA-sequencing applications. Methods Mol. Biol. 733, 93-103. doi: 10.1007/978-1-61779-089-8_7

Cooksley, C. M., Zhang, Y., Wang, H., Redl, S., Winzer, K., and Minton, N. P. (2012). Targeted mutagenesis of the Clostridium acetobutylicum Acetone-Butanol-Ethanol fermentation pathway. Metab. Eng. 14, 630-641. doi: 10.1016/j.ymben.2012.09.001
Cusick, K., Lee, Y.-Y., Youchak, B., and Belas, R. (2012). Perturbation of FliL interferes with Proteus mirabilis swarmer cell gene expression and differentiation. J. Bacteriol. 194, 437-447. doi: 10.1128/JB.05998-11

Demain, A. L., Newcomb, M., and Wu, J. H. D. (2005). Cellulase, clostridia, and ethanol. Microbiol. Mol. Biol. Rev. 69, 124-154. doi: 10.1128/MMBR.69.1.124154.2005

Dror, T. W., Rolider, A., Bayer, E. A., Lamed, R., and Shoham, Y. (2005). Regulation of major cellulosomal endoglucanases of Clostridium thermocellum differs from that of a prominent cellulosomal xylanase. J. Bacteriol. 187, 2261-2266. doi: 10.1128/JB.187.7.2261-2266.2005

Dvortsov, I., Lunina, N., Zverlov, V., and Velikodvorskaya, G. (2010). Substratebinding properties of the family 54 module of Clostridium thermocellum Lic16A laminarinase. Mol. Biol. 44, 591-595. doi: 10.1134/S002689331004014X

Dvortsov, I., Lunina, N., Zverlov, V., and Velikodvorskaya, G. (2012). Properties of four C-terminal carbohydrate-binding modules (CBM4) of laminarinase Lic16A of Clostridium thermocellum. Mol. Biol. 46, 817-822. doi: $10.1134 /$ S0026893312060039

Ferreira, S. J., Senning, M., Sonnewald, S., Keßling, P.-M., Goldstein, R., and Sonnewald, U. (2010). Comparative transcriptome analysis coupled to X-ray CT reveals sucrose supply and growth velocity as major determinants of potato tuber starch biosynthesis. BMC Genomics 11:93. doi: 10.1186/1471-2164-11-93

Gold, N., and Martin, V. (2007). Global view of the clostridium thermocellum cellulosome revealed by quantitative proteomic analysis? J. Bacteriol. 189, 6787-6795. doi: 10.1128/JB.00882-07

Hess, V., González, J. M., Parthasarathy, A., Buckel, W., and Müller, V. (2013a). Caffeate respiration in the acetogenic bacterium Acetobacterium woodii: a coenzyme A loop saves energy for caffeate activation. Appl. Environ. Microbiol. 79, 1942-1947. doi: 10.1128/AEM.03604-12

Hess, V., Schuchmann, K., and Müller, V. (2013b). The ferredoxin: NAD+ oxidoreductase (Rnf) from the acetogen Acetobacterium woodii requires $\mathrm{Na}+$ and is reversibly coupled to the membrane potential. J. Biol. Chem. 288, 31496-31502. doi: 10.1074/jbc.M113.510255

Himmel, M. E., Ding, S. Y., Johnson, D. K., Adney, W. S., Nimlos, M. R., Brady, J. W., et al. (2007). Biomass recalcitrance: engineering plants and enzymes for biofuels production. Science 315, 804-807. doi: 10.1126/science.11 37016

Huang, W., Nadeem, A., Zhang, B., Babar, M., Soller, M., and Khatib, H. (2012). Characterization and comparison of the leukocyte transcriptomes of three cattle breeds. PLoS ONE 7:e30244. doi: 10.1371/journal.pone.0030244

Irwin, D., Leathers, T., Greene, R., and Wilson, D. (2003). Corn fiber hydrolysis by Thermobifida fusca extracellular enzymes. Appl. Environ. Microbiol. 61, 352-358. doi: 10.1007/s00253-002-1210-6

Islam, R., Cicek, N., Sparling, R., and Levin, D. (2006). Effect of substrate loading on hydrogen production during anaerobic fermentation by Clostridium thermocellum 27405. Appl. Microbiol. Biotechnol. 72, 576-583. doi: 10.1007/s00253006-0316-7

Ji, H., Gheysen, G., Denil, S., Lindsey, K., Topping, J. F., Nahar, K., et al. (2013). Transcriptional analysis through RNA sequencing of giant cells induced by Meloidogyne graminicola in rice roots. J. Exp. Bot. 64, 3885-3898. doi: $10.1093 / \mathrm{jxb} / \mathrm{ert} 219$

Jia, Y., Lisch, D. R., Ohtsu, K., Scanlon, M. J., Nettleton, D., and Schnable, P. S. (2009). Loss of RNA-dependent RNA polymerase 2 (RDR2) function causes widespread and unexpected changes in the expression of transposons, genes, and 24-nt small RNAs. PLoS Genet. 5:e1000737. doi: 10.1371/journal.pgen. 1000737

Kahel-Raifer, H., Jindou, S., Bahari, L., Nataf, Y., Shoham, Y., Bayer, E. A., et al. (2010). The unique set of putative membrane-associated anti- $\sigma$ factors in Clostridium thermocellum suggests a novel extracellular carbohydrate-sensing mechanism involved in gene regulation. FEMS Microbiol. Lett. 308, 84-93. doi: 10.1111/j.1574-6968.2010.01997.x

Kanehisa, M., Araki, M., Goto, S., Hattori, M., Hirakawa, M., Itoh, M., et al. (2008). KEGG for linking genomes to life and the environment. Nucleic Acids Res. 36, D480-D484. doi: 10.1093/nar/gkm882

Lamed, R., and Zeikus, J. (1980). Ethanol production by thermophilic bacteria: relationship between fermentation product yields of and catabolic enzyme activities in Clostridium thermocellum and Thermoanaerobium brockii. J. Bacteriol. 144, 569-578.

Langmead, B., Trapnell, C., Pop, M., and Salzberg, S. L. (2009). Ultrafast and memory-efficient alignment of short DNA sequences to the human genome. Genome Biol. 10:R25. doi: 10.1186/gb-2009-10-3-r25 
Lin, C., Garrett, A. S., De Kumar, B., Smith, E. R., Gogol, M., Seidel, C., et al. (2011). Dynamic transcriptional events in embryonic stem cells mediated by the super elongation complex (SEC). Genes Dev. 25, 1486-1498. doi: 10.1101/gad.2059211

Mortazavi, A., Williams, B. A., McCue, K., Schaeffer, L., and Wold, B. (2008). Mapping and quantifying mammalian transcriptomes by RNA-Seq. Nat. Methods 5, 621-628. doi: 10.1038/nmeth.1226

Nagalakshmi, U., Wang, Z., Waern, K., Shou, C., Raha, D., Gerstein, M., et al. (2008). The transcriptional landscape of the yeast genome defined by RNA sequencing. Science 320, 1344. doi: 10.1126/science.1158441

Nataf, Y., Bahari, L., Kahel-Raifer, H., Borovok, I., Lamed, R., Bayer, E. A., et al. (2010). Clostridium thermocellum cellulosomal genes are regulated by extracytoplasmic polysaccharides via alternative sigma factors. Proc. Natl. Acad Sc. U.S.A. 107, 18646-18651. doi: 10.1073/pnas.1012175107

Nataf, Y., Yaron, S., Stahl, F., Lamed, R., Bayer, E. A., Scheper, T.-H., et al. (2009). Cellodextrin and laminaribiose $\mathrm{ABC}$ transporters in Clostridium thermocellum. J. Bacteriol. 191, 203-209. doi: 10.1128/JB.01190-08

Newcomb, M., Millen, J., Chen, C.-Y., and Wu, J. D. (2011). Co-transcription of the celC gene cluster in Clostridium thermocellum. Appl. Microbiol. Biotechnol. 90, 625-634. doi: 10.1007/s00253-011-3121-x

Olson, D. G., Tripathi, S. A., Giannone, R. J., Lo, J., Caiazza, N. C., Hogsett, D. A., et al. (2010). Deletion of the Cel48S cellulase from Clostridium thermocellum. Proc. Natl. Acad. Sci. U.S.A. 107, 17727-17732. doi: 10.1073/pnas. 1003584107

Pinheiro, B., Gilbert, H., Sakka, K., Fernandes, V., Prates, J., Alves, V., et al. (2009). Functional insights into the role of novel type I cohesin and dockerin domains from Clostridium thermocellum. Biochem. J. 424, 375-384. doi: 10.1042/BJ20091152

Raman, B., McKeown, C. K., Rodriguez, M., Brown, S. D., and Mielenz, J. R. (2011). Transcriptomic analysis of Clostridium thermocellum ATCC 27405 cellulose fermentation. BMC Microbiol. 11:134. doi: 10.1186/1471-2180-11-134

Raman, B., Pan, C., Hurst, G. B., Rodriguez, M., McKeown, C. K., Lankford, P. K., et al. (2009). Impact of pretreated switchgrass and biomass carbohydrates on clostridium thermocellum ATCC 27405 cellulosome composition: a quantitative proteomic analysis. PLoS ONE 4:e5271. doi: 10.1371/journal.pone.0005271

Riederer, A., Takasuka, T. E., Makino, S.-I., Stevenson, D. M., Bukhman, Y. V., Elsen, N. L., et al. (2011). Global gene expression patterns in Clostridium thermocellum as determined by microarray analysis of chemostat cultures on cellulose or cellobiose. Appl. Environ. Microbiol. 77, 1243-1253. doi: 10.1128/ AEM.02008-10

Rydzak, T., Levin, D. B., Cicek, N., and Sparling, R. (2009). Growth phasedependant enzyme profile of pyruvate catabolism and end-product formation in Clostridium thermocellum ATCC 27405. J. Biotechnol. 140, 169-175. doi: 10.1016/j.jbiotec.2009.01.022

Rydzak, T., Levin, D. B., Cicek, N., and Sparling, R. (2011). End-product induced metabolic shifts in Clostridium thermocellum ATCC 27405. Appl. Microbiol. Biotechnol. 92, 199-209. doi: 10.1007/s00253-011-3511-0

Rydzak, T., McQueen, P., Krokhin, O., Spicer, V., Ezzati, P., Dwivedi, R., et al. (2012). Proteomic analysis of Clostridium thermocellum core metabolism: relative protein expression profiles and growth phase-dependent changes in protein expression. BMC Microbiol. 12:214. doi: 10.1186/14712180-12-214

Schut, G. J., and Adams, M. W. (2009). The iron-hydrogenase of Thermotoga maritima utilizes ferredoxin and $\mathrm{NADH}$ synergistically: a new perspective on anaerobic hydrogen production. J. Bacteriol. 191, 4451-4457. doi: 10.1128/JB. 01582-08

Stevenson, D., and Weimer, P. (2005). Expression of 17 genes in Clostridium thermocellum ATCC 27405 during fermentation of cellulose or cellobiose in continuous culture. Appl. Environ. Microbiol. 71:4672. doi: 10.1128/AEM.71.8.46724678.2005

Strobel, H., Caldwell, F., and Dawson, K. (1995). Carbohydrate transport by the anaerobic thermophile Clostridium thermocellum LQRI. Appl. Environ. Microbiol. 61, 4012-4015.

Tang, F. C., Barbacioru, C., Wang, Y. Z., Nordman, E., Lee, C., Xu, N. L., et al. (2009). mRNA-Seq whole-transcriptome analysis of a single cell. Nat. Methods 6, U377-U386. doi: 10.1038/nmeth.1315

Tatusov, R. L., Galperin, M. Y., Natale, D. A., and Koonin, E. V. (2000). The COG database: a tool for genome-scale analysis of protein functions and evolution. Nucleic Acids Res. 28, 33-36. doi: 10.1093/nar/28.1.33
Templeton, D. W., Scarlata, C. J., Sluiter, J. B., and Wolfrum, E. J. (2010). Compositional analysis of lignocellulosic feedstocks. 2. Method uncertainties. J. Agric. Food Chem. 58, 9054-9062. doi: 10.1021/jf100807b

Tucker, M. P., Farmer, J. D., Keller, F. A., Schell, D. J., and Nguyen, Q. A. (1998). Comparison of yellow poplar pretreatment between NREL digester and sunds hydrolyzer. Appl. Biochem. Biotechnol. 70-72, 25-35. doi: 10.1007/BF02920121

van der Lelie, D., Taghavi, S., McCorkle, S. M., Li, L.-L., Malfatti, S. A., Monteleone, D., et al. (2012). The metagenome of an anaerobic microbial community decomposing poplar wood chips. PLoS ONE 7:e36740. doi: 10.1371/journal.pone. 0036740

Vinzant, T. B., Ponfick, L., Nagle, N. J., Ehrman, C. I., Reynolds, J. B., and Himmel, M. E. (1994). SSF comparison of selected woods from southern sawmills. Appl. Biochem. Biotechnol. 45, 611-626. doi: 10.1007/BF02941834

Wang, Z., Gerstein, M., and Snyder, M. (2009). RNA-Seq: a revolutionary tool for transcriptomics. Nature Reviews Genetics 10, 57-63. doi: 10.1038/nrg2484

Wei, H., Tucker, M. P., Baker, J. O., Harris, M., Luo, Y., Xu, Q., et al. (2012). Tracking dynamics of plant biomass composting by changes in substrate structure, microbial community, and enzyme activity. Biotechnol. Biofuels 5:20 doi: 10.1186/1754-6834-5-20

Wei, H., Xu, Q., Taylor, L., Baker, J., Tucker, M., and Ding, S. (2009). Natural paradigms of plant cell wall degradation. Curr. Opin. Biotechnol. 20, 330-338. doi: 10.1016/j.copbio.2009.05.008

Wilhelm, B. T., Marguerat, S., Watt, S., Schubert, F., Wood, V., Goodhead, I., et al. (2008). Dynamic repertoire of a eukaryotic transcriptome surveyed at singlenucleotide resolution. Nature 453, 1239-1243. doi: 10.1038/nature07002

Wilson, C. M., Rodriguez, M. Jr., Johnson, C. M., Martin, S. L., Chu, T. M., Wolfinger, R. D., et al. (2013a). Global transcriptome analysis of Clostridium thermocellum ATCC 27405 during growth on dilute acid pretreated Populus and switchgrass. Biotechnol. Biofuels 6:179. doi: 10.1186/1754-6834-6-179

Wilson, C. M., Yang, S., Rodriguez, M. Jr., Ma, Q., Johnson, C. M., Dice, L., et al. (2013b). Clostridium thermocellum transcriptomic profiles after exposure to furfural or heat stress. Biotechnol. Biofuels 6:131. doi: 10.1186/1754-6834-6-131

Yang, S., Giannone, R., Dice, L., Yang, Z., Engle, N., Tschaplinski, T., et al. (2012). Clostridium thermocellum ATCC27405 transcriptomic, metabolomic and proteomic profiles after ethanol stress. BMC Genomics 13:336. doi: 10.1186/14712164-13-336

Yang, S., Guarnieri, M. T., Smolinski, S., Ghirardi, M., and Pienkos, P. T. (2013). De novo transcriptomic analysis of hydrogen production in the green alga Chlamydomonas moewusii through RNA-Seq. Biotechnol. Biofuels 6:118. doi: 10.1186/1754-6834-6-118

Zverlov, V. V., Kellermann, J., and Schwarz, W. H. (2005). Functional subgenomics of Clostridium thermocellum cellulosomal genes: identification of the major catalytic components in the extracellular complex and detection of three new enzymes. Proteomics 5, 3646-3653. doi: 10.1002/pmic.200401199

Zverlov, V. V., and Schwarz, W. H. (2006). "The Clostridium thermocellum cellulosome: novel components and insights from the genomic sequence," in Cellulosome, eds I. Kataeva and L. Ljungdahl (New York, NY: Nova Science Publishers), 119-151.

Conflict of Interest Statement: The authors declare that the research was conducted in the absence of any commercial or financial relationships that could be construed as a potential conflict of interest.

Received: 21 November 2013; accepted: 19 March 2014; published online: 11 April 2014.

Citation: Wei H, Fu Y, Magnusson L, Baker JO, Maness P-C, Xu Q, Yang S, Bowersox A, Bogorad I, Wang W, Tucker MP, Himmel ME and Ding S-Y (2014) Comparison of transcriptional profiles of Clostridium thermocellum grown on cellobiose and pretreated yellow poplar using RNA-Seq. Front. Microbiol. 5:142. doi: 10.3389/fmicb. 2014.00142

This article was submitted to Microbial Physiology and Metabolism, a section of the journal Frontiers in Microbiology.

Copyright (C) 2014 Wei, Fu, Magnusson, Baker, Maness, Xu, Yang, Bowersox, Bogorad, Wang, Tucker, Himmel and Ding. This is an open-access article distributed under the terms of the Creative Commons Attribution License (CC BY). The use, distribution or reproduction in other forums is permitted, provided the original author(s) or licensor are credited and that the original publication in this journal is cited, in accordance with accepted academic practice. No use, distribution or reproduction is permitted which does not comply with these terms. 